\title{
Momentum Budget Evolution of Typhoon Nari (2001) During the Landfall Process
}

\author{
Ming-Jen Yang ${ }^{1, *}$, Tai-Chi Chen Wang ${ }^{1}$, Yi Zhang ${ }^{2}$, and Ching-Yi Weng ${ }^{1}$ \\ ${ }^{1}$ Department of Atmospheric Sciences, National Central University, Jhongli, Taiwan \\ ${ }^{2}$ Key Laboratory of Mesoscale Severe Weather of Ministry of Education, School of Atmospheric Sciences, \\ Nanjing University, Nanjing, China
}

Received 27 January 2011, accepted 31 May 2011

\begin{abstract}
In order to understand the evolution of tangential and radial flows of Typhoon Nari (2001) during its landfall in Taiwan, absolute angular momentum (AAM) and radial wind budgets of Nari are conducted by analyzing the MM5 simulation results with high spatial and temporal resolutions (2-km horizontal grid spacing and 2-min output interval). The AAM is nearly conserved outside the eyewall and above the boundary layer while Nari is over the ocean; after landfall, the enhanced surface friction and turbulent mixing produces mostly negative local tendencies of AAM above terrain. For the landfall storm, both the radar observation and model simulation indicate that the radial inflows at lower levels become thicker and stronger over land, and the sloping radial outflow jet is maximized at the midlevel above rugged topography. The midlevel radial outflows result from supergradient accelerations of $20-35 \mathrm{~m} \mathrm{~s}^{-1} \mathrm{~h}^{-1}$ and supergradient winds of $5-9 \mathrm{~m} \mathrm{~s}^{-1}$. The enhanced imbalance accelerations imply that the gradient wind balance is no longer appropriate to describe tangential winds over terrain. Near the eyewall, the supergradient winds can be as strong as $9 \mathrm{~m} \mathrm{~s}^{-1}$ above terrain and subgradient winds up to $-21 \mathrm{~m} \mathrm{~s}^{-1}$ are found at the surface on the lee side. The stronger force imbalances of the landfall Nari produce larger local changes of AAM and radial momentum, leading to more quickly-evolved vortex flows and secondary circulations over Taiwan's steep terrain.
\end{abstract}

Key words: Landfall typhoon, Momentum budget, Supergradient wind

Citation: Yang, M.J., T. C. Chen Wang, Y. Zhang, and C. Y. Weng, 2011: Momentum budget evolution of Typhoon Nari (2001) during the landfall process. Terr. Atmos. Ocean. Sci., 22, 595-612, doi: 10.3319/TAO.2011.05.31.01(TM)

\section{INTRODUCTION}

Although there have been many observational and modeling studies of tropical cyclones (TCs), our understanding on the evolution of TC's tangential and radial flows during the landfall process is rather limited. Gradient-wind balance (GWB) assumption for the tangential wind has been widely used in theoretical studies of TCs (Shapiro and Willoughby 1982; Emanuel 1986); however, whether the GWB model is valid to describe TC's tangential winds at mid-levels over terrain remains an open question.

Both sounding observations from La Seur and Hawkins (1963) and aircraft measurements from Willoughby (1990, 1991) found that the GWB model is a good approximation to the azimuthally-averaged tangential winds in the inner-core region for flows above the marine boundary layer (MBL)

\footnotetext{
* Corresponding author

E-mail:mingjen@ncu.edu.tw
}

and below the upper outflow layer. On the other hand, Gray and Shea (1973) documented systematic gradient-wind unbalanced flows in the eyewall, especially near the radius of maximum wind (RMW).

Zhang et al. (2001) (hereafter referred to as ZLY) performed absolute angular momentum (AAM) and radial momentum budgets, using their high-resolution simulation result (with horizontal grid spacing of $6 \mathrm{~km}$ ) of Hurricane Andrew (Liu et al. 1997), and have reconciled the controversy between Gray and Shea (1973) and Willoughby (1990). ZLY indicated that their conclusions are both correct for the levels of observations they analyzed, because of significant differences in the agradient forces and flows between the layers near the top of the MBL and the layers above. However, these studies are only for oceanic TCs, and it remains unknown whether the GWB model is still applicable when a TC makes landfall. 
As indicated by ZLY, the GWB model for the primary circulation is important for understanding TC's secondary circulation. The gradient wind imbalance is required to determine the structure of secondary circulation, which would in turn cause an intensity change of the primary circulation. Radial inflows (outflows) increase (decrease) the magnitude of tangential winds in the eyewall under the constraint of AAM conservation. On the other hand, the radial outflow in the eyewall plays an important role in transporting the air mass from the inner-core region outward to reduce the central pressure, strengthen the storm, and then intensify the tangential wind (Liu et al. 1999). While the TC vortex flow over the ocean has been extensively studied in the context of balanced dynamics, it is still uncertain how the unbalanced forces and flows are generated and distributed in the inner-core region as a TC encounters mountainous topography, such as the Central Mountain Range (CMR) in Taiwan.

Typhoon Nari struck Taiwan on 16 September 2001; it brought heavy rainfall (with three-day-total rainfall of more than $1400 \mathrm{~mm}$ ), strong wind gusts, flash flood, and caused severe economical and societal damage, including the loss of 92 lives (Sui et al. 2002). The precipitation efficiency of Typhoon Nari over the ocean was discussed in Sui et al. (2005), and the flooding simulation of Nari was examined in Li et al. (2005). Yang et al. (2008) (hereafter referred to as Part I) conducted a quadruply nested-grid MM5 simulation with the finest grid size of $2 \mathrm{~km}$ for Nari and showed that the model reproduced the kinematic and precipitation features as well as the structural changes of Nari reasonably well, as verified against radar and rain-gauge observations. These included the storm track, sizes and contraction of the eye and eyewall, spiral rainbands, rapid pressure rise $\left(\sim 1.67 \mathrm{hPa} \mathrm{h}^{-1}\right)$ during landfall, and the nearly constant storm intensity after landfall. In addition, the model captured the rainfall distribution and some local maxima associated with Taiwan's orography. Yang et al. (2011a) (hereafter referred to as Part II) further examined the structure changes and terrain-induced storm asymmetries after Nari's landfall in Taiwan by comparing the full-terrain control run with the reduced-terrain sensitivity simulations.

In order to further understand the evolution of tangential and radial flows of Typhoon Nari upon its landfall in Taiwan, AAM and radial wind budgets of the storm are conducted in this study by analyzing the MM5 output from Part I with high spatial and temporal resolutions (2-km horizontal grid spacing and 2-min output interval). Before landfall, Nari's tangential wind exhibited an evident wavenumber-1 signature, with radial inflow at lower levels and outflow at upper levels (see Figs. 12 and 16 of Part I). After landfall, the horizontal winds became highly asymmetric (see Figs. 13 and 15 of Part I). Both radar observation (Figs. 1c, e) and MM5 simulation (Figs. 1d, f) indicate that the radial inflow at lower levels became thicker and stronger over land than that off shore, and the sloping radial outflow jet was maximized at the midlevel above the rugged terrain. Note that the simulated stronger radial wind, compared to the radar-derived radial wind, is consistent with the more intense storm intensity in the simulation (see Fig. 5 of Part I). The time difference between Figs. 1a and b is due to the 3-h earlier landfall time of the simulated storm, and the slightly different orientation of the southwest sectors between the observation and simulation is to adjust for the different terrain imposed on the simulated storm due to the landfall position error.

The first objective of this study is to investigate the evolution of tangential and radial flows of Nari during its landfall in Taiwan, especially for the transition from the more axisymmetric structures over the ocean to the asymmetric features over mountains. The second objective is to understand the extent that the GWB is still valid for a TC making landfall. The third purpose is to examine the physical mechanism responsible of the sloping midlevel radial outflow over topography. Through analyses of the AAM and radial momentum budgets, we hope to gain insight into how the balanced and unbalanced flows are generated and azimuthally distributed as Nari encountered the mountainous topography in Taiwan.

\section{METHODOLOGY}

All momentum budget calculations are based on the governing equations used in the PSU-NCAR MM5 model (Dudhia 1993; Grell et al. 1995). Following ZLY, because of the axisymmetric nature of TCs, we also discuss the evolution of tangential and radial flows of Nari in the cylindrical coordinate $(r, \lambda, z)$, where $r$ is the radius from the TC center pointing outward, $\lambda$ is the azimuthal angle, and $z$ is the vertical height.

The governing equation for the radial wind in the cylindrical coordinate can be written as

$$
\frac{d U}{d t}=-\frac{1}{\rho} \frac{\partial p}{\partial r}+\frac{V^{2}}{r}+f V-2 \Omega \cos \phi W \cos \lambda+U_{T B}
$$

where

$\frac{d}{d t}=\frac{\delta}{\delta t}+U^{\prime} \frac{\partial}{\partial r}+\frac{V^{\prime}}{r} \frac{\partial}{\partial \lambda}+W \frac{\partial}{\partial z}$

$W, U$, and $V$ are the vertical, radial and tangential winds in the cylindrical coordinate; $\Omega$ is the angular velocity of the earth; $\phi$ is the latitude. To separate the translation effect from storm's dynamical processes, we define $\delta / \delta t=\partial / \partial t+\mathbf{C} \cdot \nabla$ as the local tendency, where $\mathbf{C}$ is the TC's translation vector, and $U^{\prime}$ and $V^{\prime}$ are the storm-relative radial and tangential winds, respectively. Equation (1) 

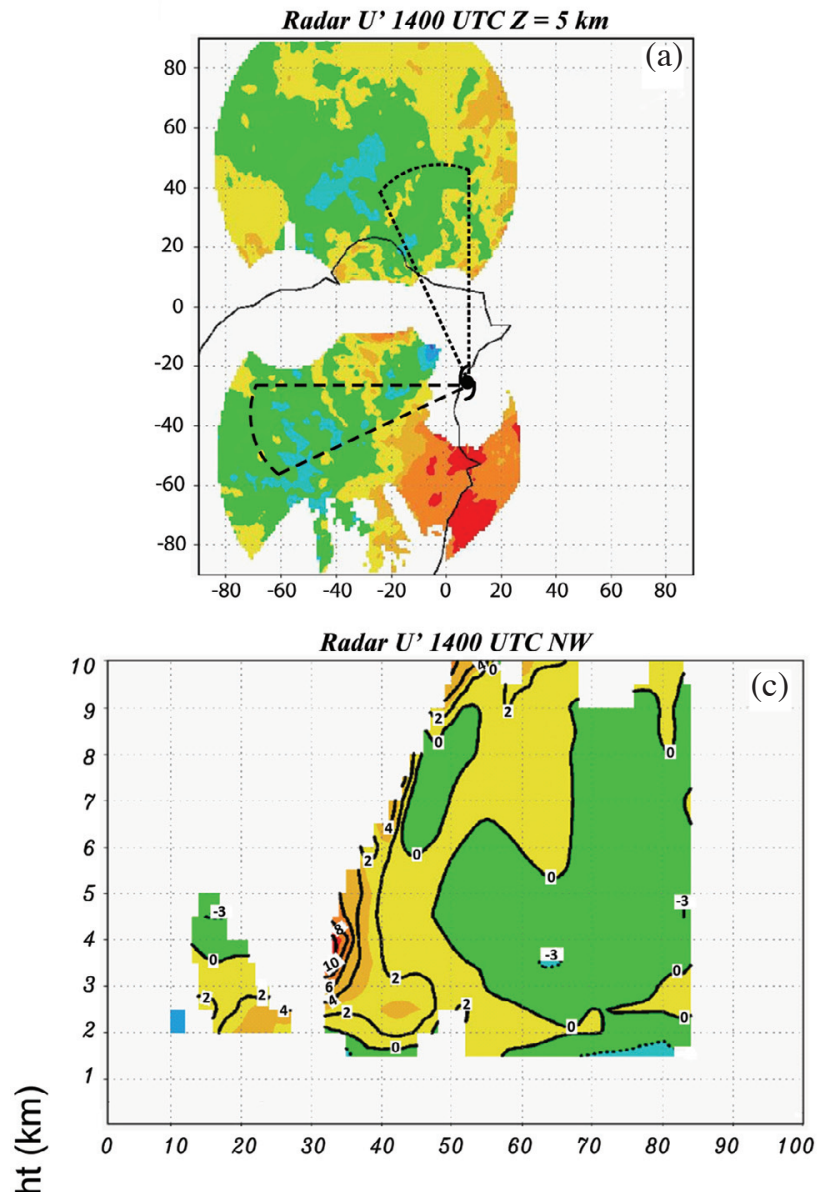

(c)
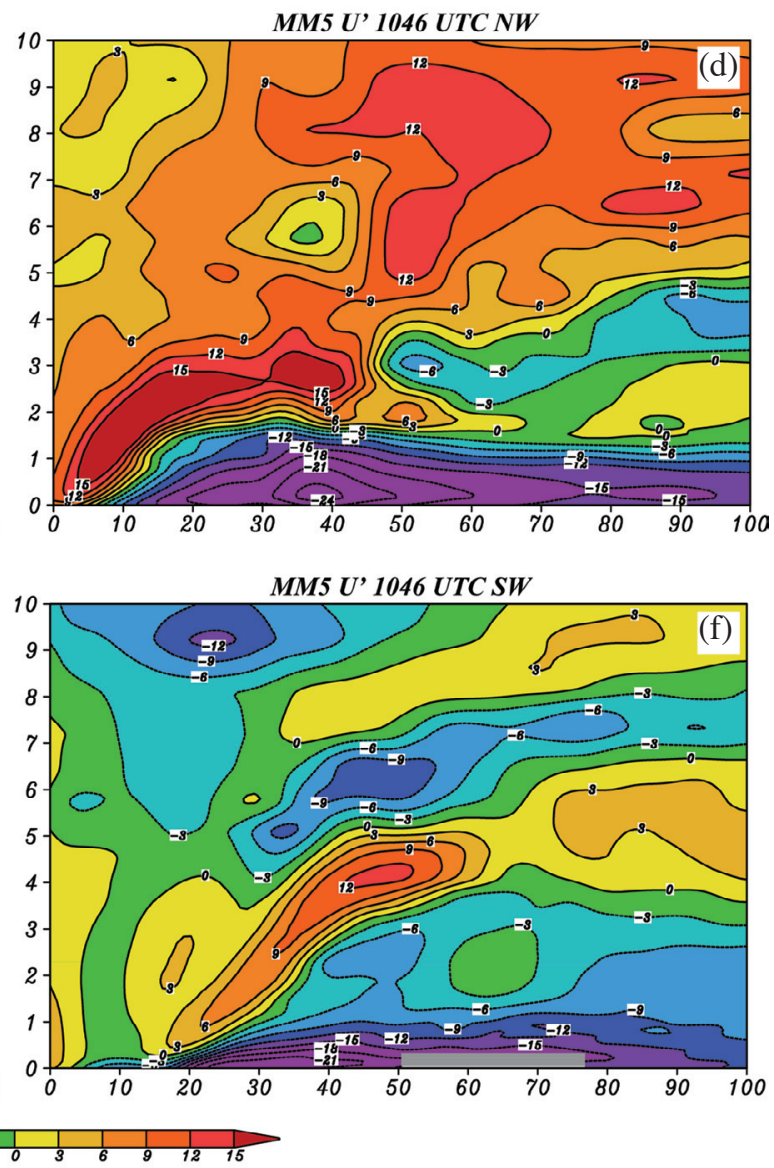

Radius (km)

Fig. 1. (a) Radar-observed radial wind ( $\mathrm{m} \mathrm{s}^{-1}$; relative to TC center) at 5-km level at 1400 UTC 16 September and (b) MM5-simulated radial wind at 1046 UTC 16 September 2001; vertical structures of (c) the observed and (d) simulated radial wind averaged in the dotted NW sector, and (e) the observed and (f) simulated radial wind averaged in the dashed SW sector. The horizontal positions of the dotted NW and dashed SW sectors are indicated in (a) and (b).

states that the Lagrangian radial acceleration is determined by the radial pressure gradient force $\left(\mathrm{PGF}_{\mathrm{R}} ; U_{P}\right)$, the centrifugal force $\left(U_{E}\right)$, the Coriolis force $\left(U_{C}\right)$ including the effects of vertical motion, and the effects of the PBL and turbulent mixing $\left(U_{T B}\right)$. Note that the sign error for the Coriolis effect by vertical motion in ZLY has been corrected in Eq. (1).

The governing equation for the tangential wind in the cylindrical coordinate can be written as 
$\frac{d V}{d t}=-\frac{1}{\rho} \frac{\partial p}{r \partial r}-\frac{U V}{r}-f U+2 \Omega \cos \phi W \sin \lambda+V_{T B}$

As in ZLY, it is more insightful to rewrite Eq. (3) in terms of the AAM defined by

$M=r\left(V+\frac{f r}{2}\right)$

with the form

$\frac{d M}{d t}=-\frac{1}{\rho} \frac{\partial p}{\partial \lambda}-2 r \Omega \cos \phi W \sin \lambda+\frac{r^{2}}{2} v_{m} \beta+r V_{T B}$

where $\beta$ is the variation of the Coriolis parameter with latitude and $v_{m}$ represents the projections of $U$ and $V$ onto the longitudinal axes. Equation (5) indicates that the Lagrangian derivative of AAM results from a pressure torque, a longitudinally-oriented Coriolis torque by the vertical motion, a beta torque, and a torque due to the PBL effect and turbulent mixing process. Note that the sign error for the longitudinally-oriented Coriolis torque by the vertical motion in ZLY has been corrected in Eq. (5).

For Typhoon Nari, all momentum budget terms above are obtained from the MM5 output on the finest $2-\mathrm{km}$ grid at 2-min intervals from the $13-14$-h (valid at $0100-0200$ UTC 16 September) and 22 - 23-h (valid at $1000-1100$ UTC 16 September 2001) integrations, respectively. For the simulation period of $13-14 \mathrm{~h}$, which is $8-9 \mathrm{~h}$ prior to landfall, the storm is still intensifying (see Fig. 5 of Part I). For the simulation period of $22-23 \mathrm{~h}$, it is the first onehour period after storm's landfall (the simulated Nari makes landfall on Taiwan at $t=22 \mathrm{~h}$ or 1000 UTC 16 September 2001; see Fig. 4 of Part I). Each variable is transformed from the MM5 coordinate $(x, y, \sigma)$ into the cylindrical coordinate $(r, \lambda, z)$ with the typhoon center at the origin. When Nari was over the ocean, the TC center is defined as the center of minimum sea-level pressure; thus, when Nari was over northern Taiwan, the TC center is defined as the primary vortex circulation center at 4-km altitude (above the highest peak of the Snow Mountain Range). The earth-relative local tendency term $(\partial / \partial t)$ is calculated using the second-order central difference with a time interval of $2 \mathrm{~min}$. The terms for PBL effect and turbulent mixing process $\left(U_{T B}\right.$ and $\left.V_{T B}\right)$ are calculated as the residues from other terms in Eqs. (1) and (3); thus some numerical errors are included in these terms as well. For axisymmetric results presented in the next section, each budget term is averaged azimuthally and temporally over the one-hour period. The horizontal winds relative to the storm are obtained by subtracting out the storm's translation vector at an east-northeasterly speed of $3.89 \mathrm{~m} \mathrm{~s}^{-1}$ at the 13 - 14-h period and a northeasterly speed of $3.06 \mathrm{~m} \mathrm{~s}^{-1}$ at the $22-23$-h period, respectively (see Table 1 and Fig. 4 of Part I).

\section{AXISYMMETRIC AAM AND RADIAL MOMEN- TUM BUDGETS OVER OCEAN}

To understand the velocity and AAM features of the oceanic Typhoon Nari, Fig. 2 shows the NE-SW (alongtrack) $A_{1} B_{1}$ vertical cross sections of the temporally-averaged $(13-14 \mathrm{~h})$ vertical velocity $(W)$, radial $\left(U^{\prime}\right)$ and tangential $\left(V^{\prime}\right)$ flows, and AAM of the storm (see Fig. 10 of Part I for the horizontal position of $\mathrm{A}_{1} \mathrm{~B}_{1}$ cross section). In general, Fig. 2 displays the typical kinematic and AAM
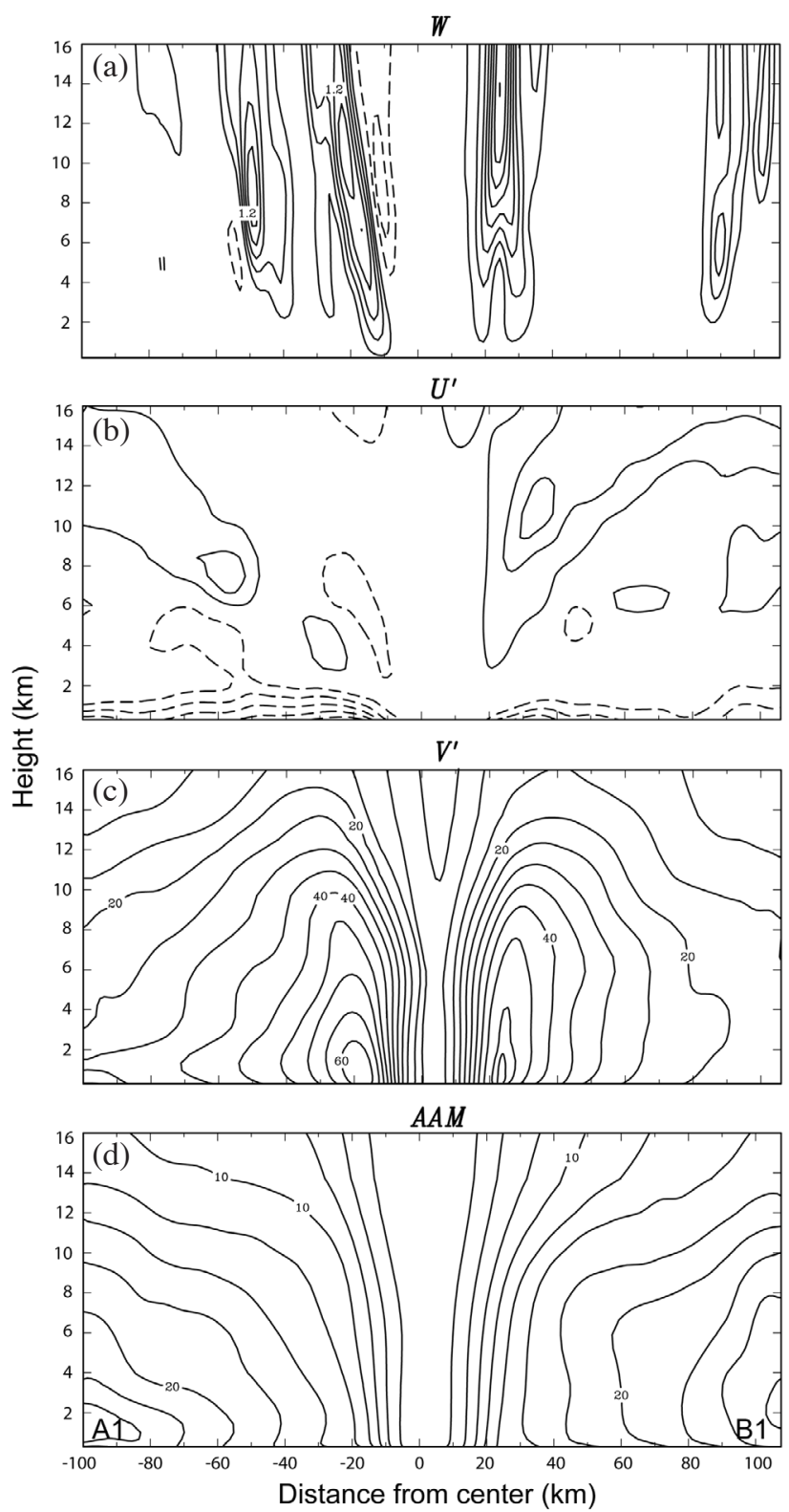

Fig. 2. Along-track vertical cross sections of the hourly-averaged (a) vertical velocity $(W)$ at intervals of $0.25 \mathrm{~m} \mathrm{~s}^{-1}$, (b) radial winds $\left(U^{\prime}\right)$ at $2.5 \mathrm{~m} \mathrm{~s}^{-1},(\mathrm{c})$ tangential winds $\left(V^{\prime}\right)$ at intervals of $5 \mathrm{~m} \mathrm{~s}^{-1}$, and (d) absolute angular momentum (AAM) at intervals of $2.5 \times 10^{5} \mathrm{~m}^{2} \mathrm{~s}^{-1}$ of Typhoon Nari over the ocean at the period of 0100 - 0200 UTC 16 September $2001(t=13-14 \mathrm{~h})$. Horizontal winds at (b) and (c) are storm-relative, and zero contours in (a) and (b) are omitted. 
features of an oceanic TC at its developing stage, and some degree of asymmetry induced by storm's internal dynamical processes can be seen. For example, eyewall updraft in the northeast quadrant (right half of Fig. 2a) is stronger and more upright than that in the southwest quadrant (left half of Fig. 2a); the low-level inflow (upper-level outflow) is stronger and deeper (weaker and shallower) in the southwest quadrant than that in the northeast quadrant (Fig. 2b). The tangential wind and AAM field, on the other hand, show a less degree of asymmetry on storm structure (Figs. 2c, d).

Figure 3 displays corresponding temporally- and azimuthally-averaged kinematics and AAM fields of the oceanic Nari. The axisymmetric tangential flow exhibits intense flow $\left(V_{\max }^{\prime}=57 \mathrm{~m} \mathrm{~s}^{-1}\right)$ maximized at $r=20 \mathrm{~km}$ at the top of MBL (Fig. 3c). The axis of RMW lies slightly outside the slantwise updrafts (labeled as UP in Fig. 3a) in the eyewall and slopes outward with height up to $12 \mathrm{~km}$. The surface friction causes strong vertical wind shear inside the MBL (for levels below $1-1.5 \mathrm{~km}$ in Fig. 3c). The low-level inflow, peaked $\left(>14 \mathrm{~m} \mathrm{~s}^{-1}\right)$ at the surface near $r=30 \mathrm{~km}$, turns sharply upward into the deep updrafts in the eyewall (Fig. 3a) and the outflow jet $\left(>6 \mathrm{~m} \mathrm{~s}^{-1}\right)$ at upper levels peaked near $12 \mathrm{~km}$ (Fig. 3b). In the outer rainband region $(r>50 \mathrm{~km})$, there are midlevel radial inflows sloping downward to the inner-core region, associated with the latent cooling by sublimation and melting [see Fig. 7c of Yang et al. (2011b)]. Sloping downdrafts (labeled as DN in Fig. 3a) occur at the interface between the eye and eyewall. The axisymmetric AAM shows significant inward decreases with a large vertical gradient in the MBL by surface friction and outward increases in upper outflow layers by radial advection (Fig. 3d). The secondary-circulation flow vectors follow closely the AAM surfaces within the eyewall $(r=$ $10-30 \mathrm{~km}$ ).
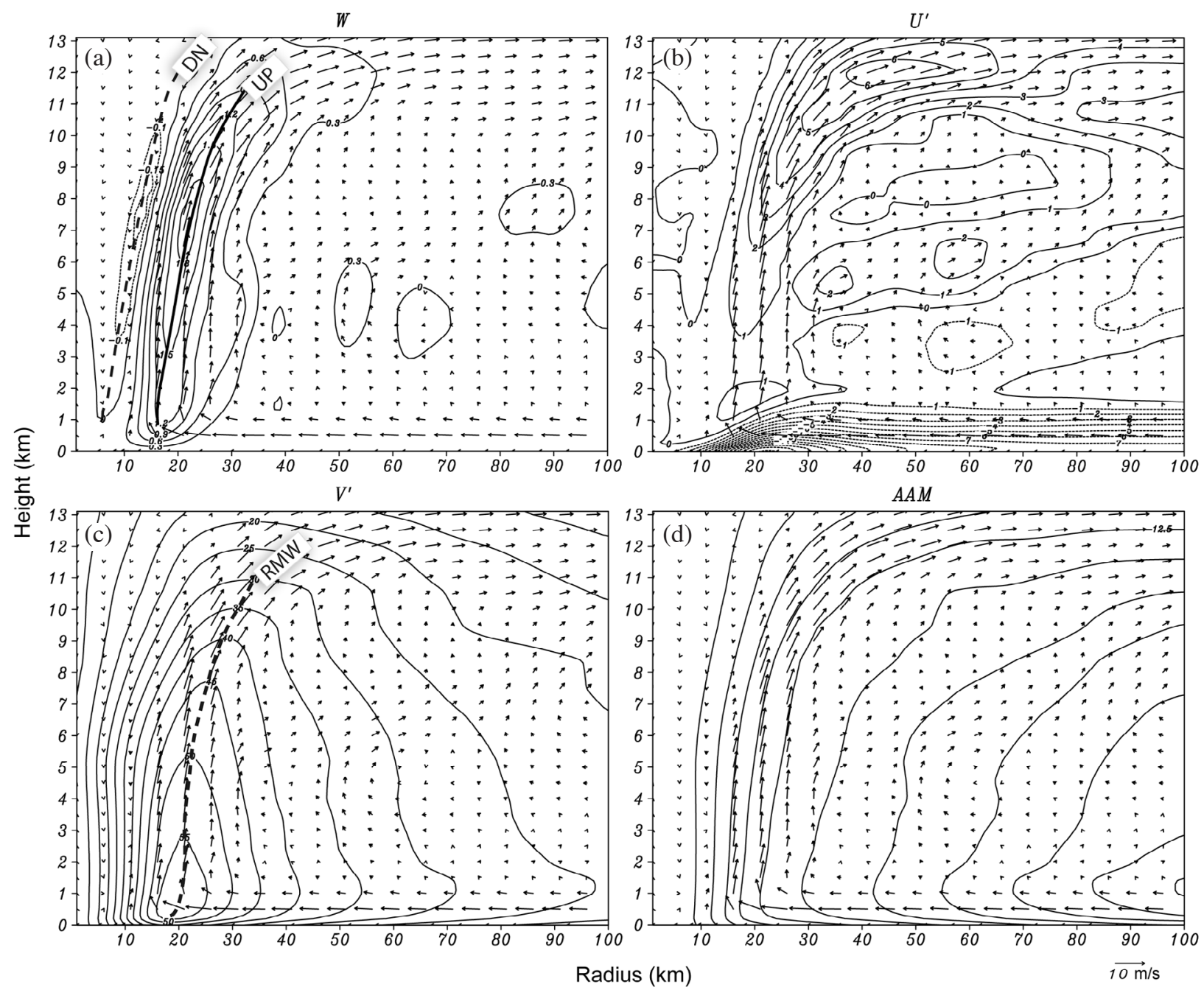

Fig. 3. Radius-height cross sections of the hourly- and azimuthally-averaged (a) vertical velocity $(W)$ at intervals of $0.3 \mathrm{~m} \mathrm{~s}^{-1}$ for updrafts with extra contours at $-0.2,-0.15,-0.1,-0.5 \mathrm{~m} \mathrm{~s}^{-1}$, (b) radial winds $\left(U^{\prime}\right)$ at $1 \mathrm{~m} \mathrm{~s}^{-1}$, (c) tangential winds $\left(V^{\prime}\right)$ at intervals of $5 \mathrm{~m} \mathrm{~s}^{-1}$, and (d) absolute angular momentum (AAM) at intervals of $2.5 \times 10^{5} \mathrm{~m}^{2} \mathrm{~s}^{-1}$ of Typhoon Nari over the ocean, superposed with the cross-sectional flow vectors, taken from the 13 - $14 \mathrm{~h}$ integration or the period of 0100 - 0200 UTC 16 September 2001. Horizontal winds at (b) and (c) are storm-relative. The radius of maximum wind, the axes of the eyewall updrafts and the inner-edge downdrafts are represented by RMW, UP, and DN, respectively. 
Figure 4 shows the hourly-averaged axisymmetric AAM budget terms of the oceanic Typhoon Nari, which are similar to those in Hurricane Andrew (see Fig. 2 of ZLY). The Lagrangian tendency $(d M / d t$; Fig. 4a) represents the sources/sinks of AAM associated with the turbulent mixing and surface friction (mainly within the MBL), since the pressure torque almost vanishes after the azimuthal averaging and the other two terms on the right-hand side of Eq. (5) are very small. Above the MBL, turbulent mixing decreases the local AAM within and outside the eyewall $(r=15-60 \mathrm{~km})$ and slightly increases AAM inward to the eye. The MBL (below $1-2 \mathrm{~km}$ ) is a sink of the AAM as the result of frictional dissipation; above the MBL and outside the region of intense turbulent mixing, the AAM is nearly conserved with very small Lagrangian tendency. The horizontal advection of the $\operatorname{AAM}\left(M_{H} ;\right.$ Fig. $\left.4 \mathrm{~b}\right)$ is opposite in sign to the vertical advection of the AAM $\left(M_{V} ;\right.$ Fig. $\left.4 \mathrm{c}\right)$ at the inner edge of eyewall $(r=10-20 \mathrm{~km})$, as expected from the conservation of the AAM. A similar out-of-phase relationship between the horizontal and vertical advections of horizontal momentum is also noted by Yang and Houze (1996) (see their Fig. 10) for deep convection within a squall-line system. The radial inflow in the MBL increases the AAM of the storm by transporting the high-AAM air in the outer region inward (Fig. 4b), and the vertical advection further transports the high-AAM air upward in the eyewall and outward in the upper outflow layer (Fig. 4c). The net result is that the local AAM tendency above the MBL is a small residue between horizontal and vertical advections (Fig. 4d). The updraft axis in the eyewall (labeled by UP) corresponds to negative AAM tendency, indicating a slow spindown of tangential flow inside the eyewall at a later time.

Figure 5 illustrates all the radial-momentum budget terms in Eq. (1) and the extent of different balanced-wind approximations when Nari is still over the ocean. The most intense $\mathrm{PGF}_{\mathrm{R}}\left(U_{P}\right)$ occurs inside the eyewall and its axis fol-

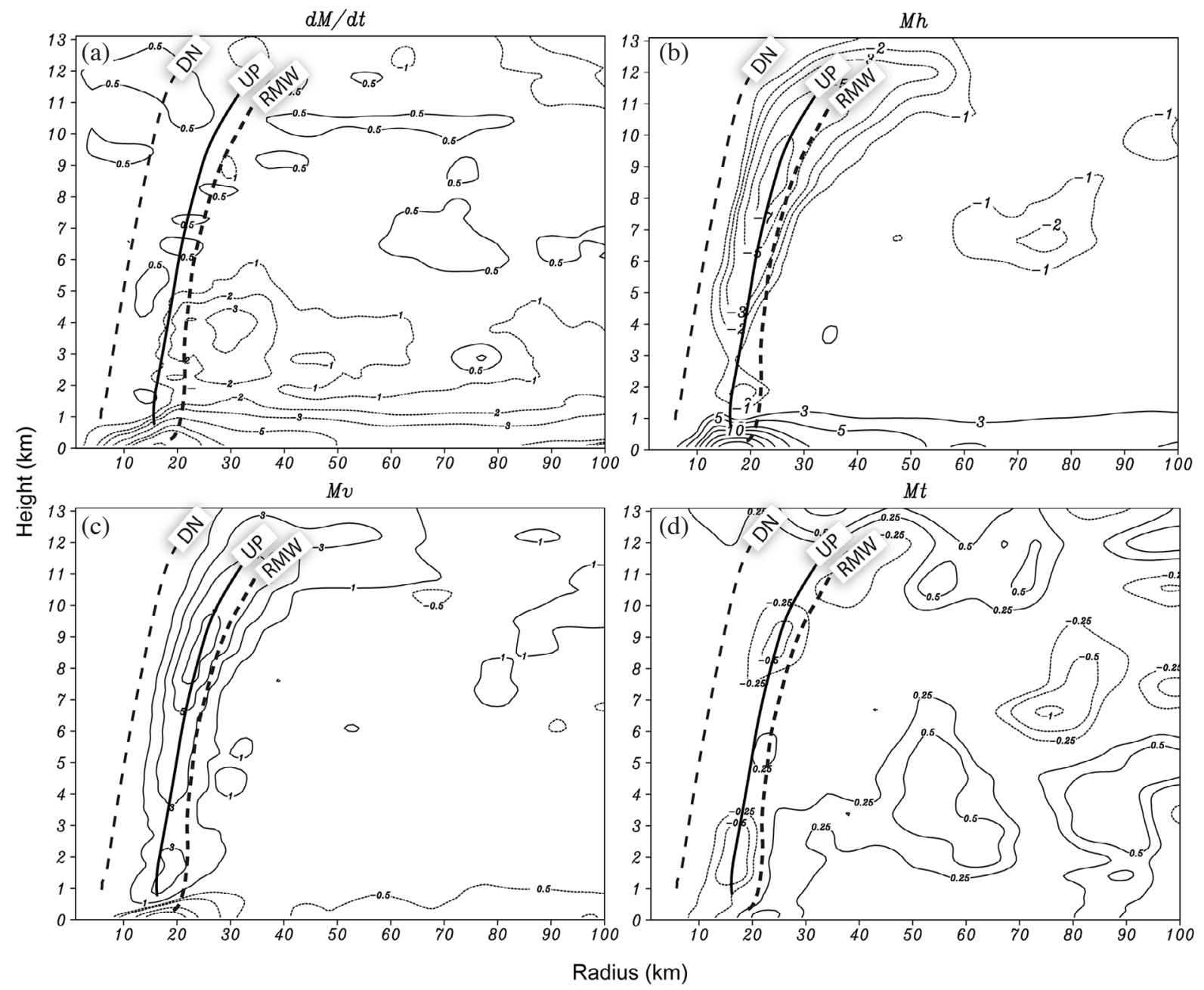

Fig. 4. As in Fig. 3 but for the hourly- and azimuthally-averaged AAM budget of Typhoon Nari: (a) the net Lagrangian tendency due to all the sources/sinks $(d M / d t)$, (b) the horizontal advection $\left(M_{H}\right)$, (c) the vertical advection $\left(M_{V}\right)$, and (d) the local tendency $\left(M_{t}\right)$. (a) is contoured at $0.5, \pm 1$, $-2,-3,-5,-8,-10$, and $-16 \times 10^{5} \mathrm{~m}^{2} \mathrm{~s}^{-1} \mathrm{~h}^{-1}$; (b) is contoured at $-1,-2, \pm 3, \pm 5,-7,-9, \pm 8, \pm 10, \pm 13, \pm 16$, and $\pm 20 \times 10^{5} \mathrm{~m}^{2} \mathrm{~s}^{-1} \mathrm{~h}^{-1}$; (c) is contoured at -0.5 , $\pm 1, \pm 3, \pm 5, \pm 10, \pm 16$, and $\pm 20 \times 10^{5} \mathrm{~m}^{2} \mathrm{~s}^{-1} \mathrm{~h}^{-1} ;(\mathrm{d})$ is contoured at $\pm 0.25, \pm 0.5$, and $\pm 1 \times 10^{5} \mathrm{~m}^{2} \mathrm{~s}^{-1} \mathrm{~h}^{-1}$. 
lows closely that of the eyewall updraft (UP; see Figs. 3a and 5a); this negative $\mathrm{PGF}_{\mathrm{R}}$ decreases rapidly with height and accounts for the generation of radial inflow in the MBL that increases toward the RMW. The centrifugal force $\left(U_{E}\right)$ has similar structure but with opposite sign to the $\mathrm{PGF}_{\mathrm{R}}$ (Fig. 5b). The sum of these two terms is the cyclostrophic force imbalance $\left(U_{P E}=U_{P}+U_{E} ;\right.$ Fig. $\left.5 \mathrm{c}\right)$. For the eye and the inner edge of the eyewall $(r<20 \mathrm{~km}), U_{E}$ overcompensates
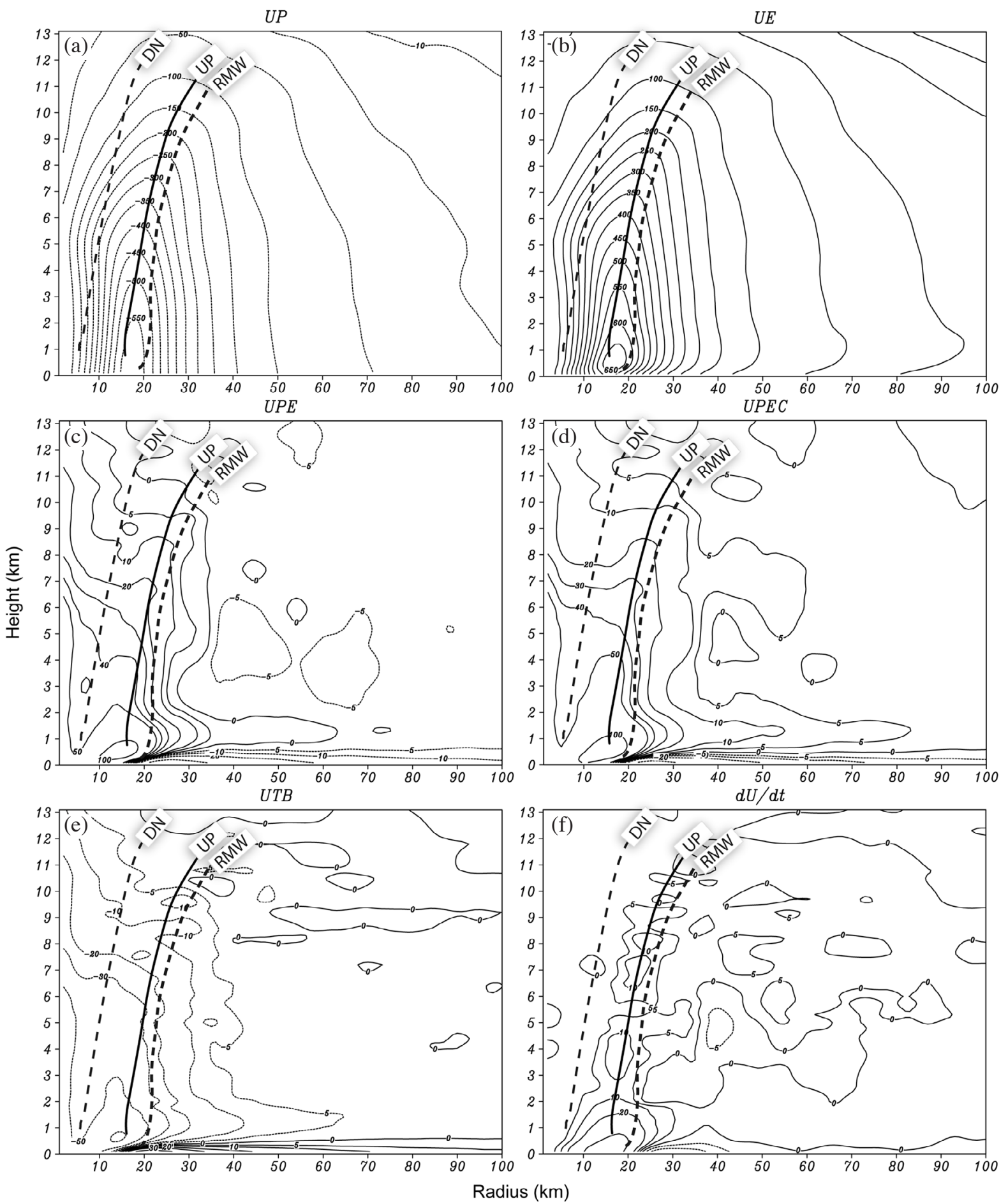

Fig. 5. As in Fig. 3 but for the hourly- and azimuthally-averaged radial momentum budget (in units of $\mathrm{m} \mathrm{s}^{-1} \mathrm{~h}^{-1}$ ) of Typhoon Nari: (a) the radial pressure gradient force $\left(\mathrm{PGF}_{\mathrm{R}}, U_{P}\right)$, (b) the centrifugal force $\left(U_{E}\right)$, (c) the cyclostrophic force imbalance $\left(U_{P E}=U_{P}+U_{E}\right)$, (d) the gradient-force imbalance $\left(U_{P E C}=U_{P E}+U_{C}\right)$, (e) the diffusion and boundary layer effects $\left(U_{T B}\right)$, and (f) the Lagrangian tendency $(d U / d t)$. Both (a) and (b) are contoured at intervals of $50 \mathrm{~m} \mathrm{~s}^{-1} \mathrm{~h}^{-1}$ with extra contours at $\pm 5, \pm 10, \pm 25 \mathrm{~m} \mathrm{~s}^{-1} \mathrm{~h}^{-1}$; both (c) and (d) are contoured at $0, \pm 5, \pm 10, \pm 20,30,40, \pm 50$, and $\pm 100 \mathrm{~m} \mathrm{~s}^{-1} \mathrm{~h}^{-1}$; (e) is contoured at $0, \pm 5, \pm 10, \pm 20, \pm 30$, and $\pm 50 \mathrm{~m} \mathrm{~s}^{-1} \mathrm{~h}^{-1}$; (f) is contoured at $0, \pm 5, \pm 10, \pm 20$, and $\pm 50 \mathrm{~m} \mathrm{~s}^{-1} \mathrm{~h}^{-1}$. 
$U_{P}$, leading to strong supercyclostrophic accelerations $\left(U_{P E}\right.$ $>0)$; conversely, small subcyclostrophic accelerations $\left(U_{P E}\right.$ $<0$ ) occur at the outer rainband region and mostly beneath the MBL. The degree of gradient-wind imbalance $\left(U_{P E C}=\right.$ $\left.U_{P E}+U_{C}\right)$ is shown in Fig. 5d. Inside the RMW, because the Coriolis acceleration $\left(U_{C}\right)$ is very small compared to $U_{\mathrm{P}}$ or $U_{E}$, the distribution of gradient-wind imbalance $\left(U_{P E C}\right)$ is very similar to that of cyclostrophic force imbalance $\left(U_{P E}\right)$. After including $U_{C}$, the positive (radially outward) accelerations are more pronounced for the flow above the MBL in the eye and eyewall, indicating significant supergradient tendencies $\left(U_{P E C}>0\right)$. The effects of $\mathrm{MBL}$ and turbulent mixing on radial winds are shown in Fig. 5e. The positive acceleration near the surface within the MBL indicates the slowdown of radial inflow by surface friction; strong turbulent mixing in the eye and eyewall $(r<30 \mathrm{~km})$ produces negative acceleration to reduce the radial outflows at the mid-to-upper levels. The Lagrangian acceleration (Fig. 5f) has similar features to those of the gradient-wind imbalance within the eyewall, and it shows positive (radially outward) accelerations at the inner edge of the eyewall and negative (radially inward) accelerations below the MBL at the outer edge of the eyewall $(r=20-40 \mathrm{~km})$.

Figure 6 shows the effects of horizontal (radial) and vertical advections in determining the radial Lagrangian tendencies of the oceanic Nari at $t=13-14 \mathrm{~h}$ integration. It is clear from Figs. $4 \mathrm{~b}$ and $6 \mathrm{a}$ that radial advection acts to slow down the low-level radial inflows in the MBL outside the eyewall $(r>25 \mathrm{~km})$ and increase radial inflows inside the eyewall $(r<25 \mathrm{~km})$; similar results are also found for Hurricane Andrew (see Fig. 8a in ZLY). In the eyewall, radial advection enhances the radial outflow in the upper troposphere (Fig. 6a), whereas vertical advection reduces the local radial outflow below the updraft axis and slightly increases it above (Fig. 6b). The local radial tendency with a peak magnitude of $2 \mathrm{~m} \mathrm{~s}^{-1} \mathrm{~h}^{-1}$ is one order of magnitude smaller than radial and vertical advections (Fig. 6c), and it is much smaller than the Lagrangian tendency (cf. Figs. $5 \mathrm{f}$ and 6c). This small local tendency suggests that the oceanic Nari's radial flows evolve very slowly during its intensify-
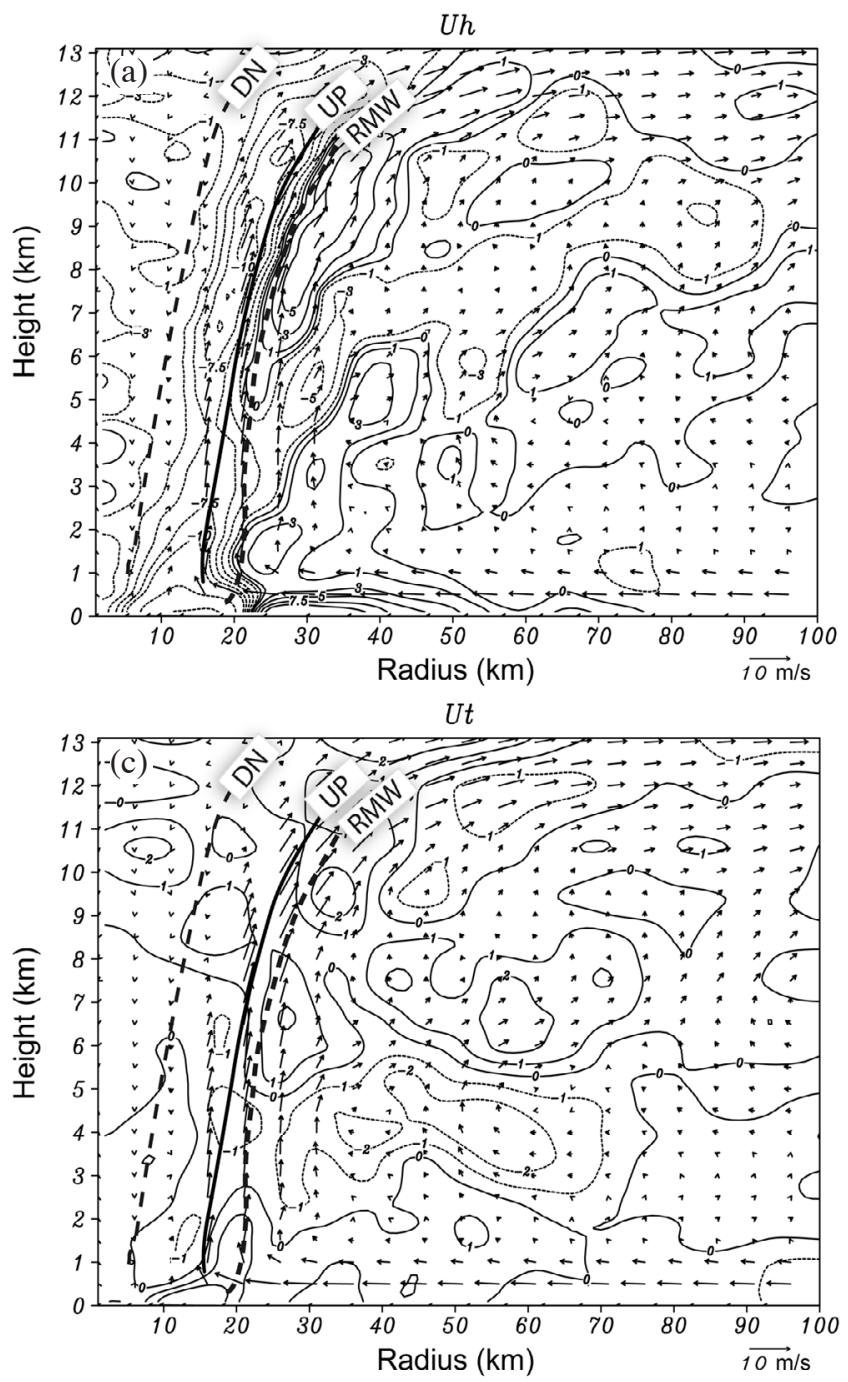

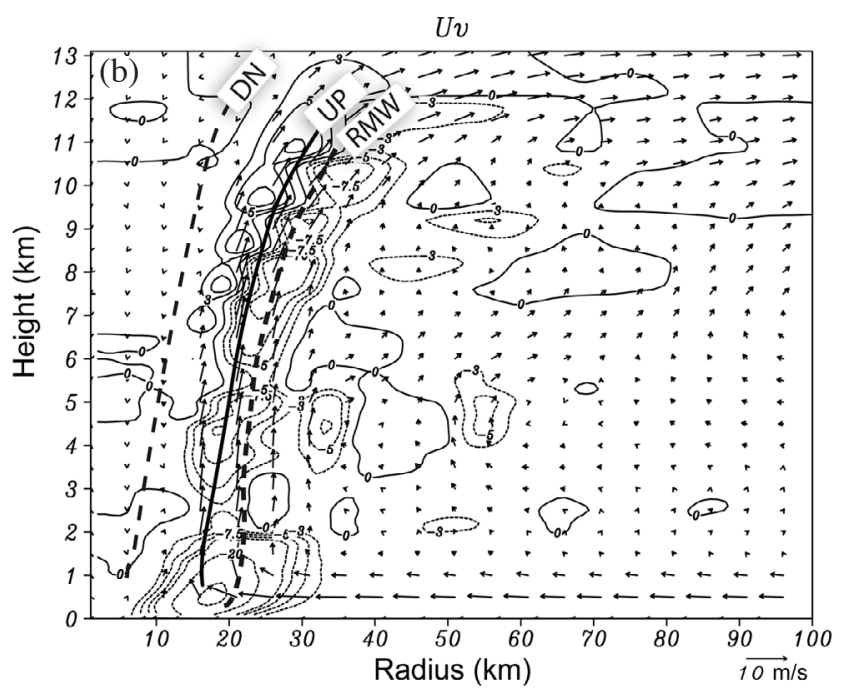

Fig. 6. As in Fig. 4 but for (a) horizontal advection $\left(U_{H}\right)$, (b) vertical advection $\left(U_{V}\right)$, and (c) local tendency $\left(U_{t}\right)$ of the radial momentum budget of Typhoon Nari taken from the $13-14 \mathrm{~h}$ integration or the period of 0100 - 0200 UTC 16 September 2001. (a) is contoured at $0, \pm 1, \pm 3, \pm 5, \pm 7.5, \pm 10$, and $\pm 20 \mathrm{~m} \mathrm{~s}^{-1} \mathrm{~h}^{-1}$; (b) is contoured at $0, \pm 3, \pm 5, \pm 7.5, \pm 10$, and $\pm 20 \mathrm{~m} \mathrm{~s}^{-1} \mathrm{~h}^{-1}$; (c) is contoured at $0, \pm 1, \pm 2, \pm 4, \pm 6$, and $\pm 10 \mathrm{~m} \mathrm{~s}^{-1} \mathrm{~h}^{-1}$. 
ing stage. The local radial tendency results in positive (outward) accelerations in the upper troposphere (indicating the strengthening of upper-level outflows) and below the MBL inside the eye (implying the radial transport of the low-level air from the eye to eyewall to further reduce the central pressure) at this developing stage.

The acyclostrophic and agradient winds of the oceanic phase of Nari are shown in Fig. 7. Supercyclostrophic winds occur inside the eyewall within the troposphere $(r<30 \mathrm{~km}$, $z<10 \mathrm{~km}$ in Fig. 7a), with a peak wind of $6 \mathrm{~m} \mathrm{~s}^{-1}$ at the inner edge of eyewall $(r \sim 15 \mathrm{~km})$ within the MBL. On the other hand, weak subcyclostrophic winds are found near the surface $(z<1 \mathrm{~km})$ outside the eyewall and outer region $(r>20 \mathrm{~km})$. Similarly, supergradient winds occur inside the eyewall with the maximum intensity of $>6 \mathrm{~m} \mathrm{~s}^{-1}$ at the low level at the inner edge of eyewall (Fig. 7b). Peak supergradient winds are also located near the top of the MBL $(z=1-1.5 \mathrm{~km})$ in the outer region. Subgradient winds mainly occur outside the eyewall $(r>20 \mathrm{~km})$ beneath the MBL.

\section{ASYMMETRIC AAM AND RADIAL MOMEN- TUM BUDGETS OVER LAND}

After the simulated storm makes landfall in northern Taiwan at $t=22 \mathrm{~h}$ (valid at 1000 UTC 16 September 2001), the steep terrain of the Snow Mountain Range (SMR; Fig. 8b) induces strong asymmetry on the storm structure. A terrain-sensitivity experiment indicates that the asymmetry is greatly reduced if Taiwan terrain is completely removed (see Figs. 10 and 11 of Part II). Figure 8a displays Nari's tangential wind at $z=3 \mathrm{~km}$ at the time of landfall. This $\mathrm{AB}$ cross section in the along-track (NE to $\mathrm{SW}$ ) direction, which encounters Mount Snow (the highest peak of SMR), is chosen to show the SMR's influence on Nari's AAM and radial-momentum budget fields.

Figure 9 clearly exhibits the temporally-averaged kinematic and AAM structures along the AB vertical cross section during the first one-hour period $(t=22-23 \mathrm{~h})$ after the storm's landfall. Although the general features of the landfall storm along the $\mathrm{AB}$ cross section in Fig. 9 are similar to those prior to landfall in the NE-SW direction in Fig. 2, there are several noticeable differences needed to be mentioned. The vertical axis of the RMW is tilted outward with a height below $4 \mathrm{~km}$ over the terrain (Fig. 9c). The low-level inflow was enhanced with s peak magnitude of $25 \mathrm{~m} \mathrm{~s}^{-1}$ off shore (Fig. 9b), due to the enhanced friction and storm retardation by Taiwan's topography (Kurihara and Bender 1982; Yeh and Elsberry 1993a, b). The outward-sloping radial outflow at the midlevel in the inner core over land is very pronounced ( $U_{\max }^{\prime}>15 \mathrm{~m} \mathrm{~s}^{-1}$ in Fig. 9b; its axis is labeled by OUT), compared to its counterpart over the ocean. This midlevel outflow jet over land is weaker and less outwardtilted if the Taiwan terrain is totally removed (see Fig. 4 of Part II). Updrafts in the eyewall over land are also outwardtilted vertically and show more cellular structures above the terrain (Fig. 9a). The AAM surfaces are tilted outward vertically in the eyewall over the terrain $(r=20-50 \mathrm{~km})$ and the "buckling height" of the AAM surface (the height for the AAM with reduced vertical gradient above in the lower levels) occurs at a higher altitude in the outer region $(r>$ $40 \mathrm{~km}$ ) over land than that over the ocean, indicating the rapid spindown of tangential flow due to the enhanced surface friction by the SMR topography (Fig. 9d).

Figure 10 shows the temporally-averaged AAM budget terms at $t=22-23 \mathrm{~h}$ along the $\mathrm{AB}$ vertical cross section. Positive Lagrangian tendencies of AAM above the CMR
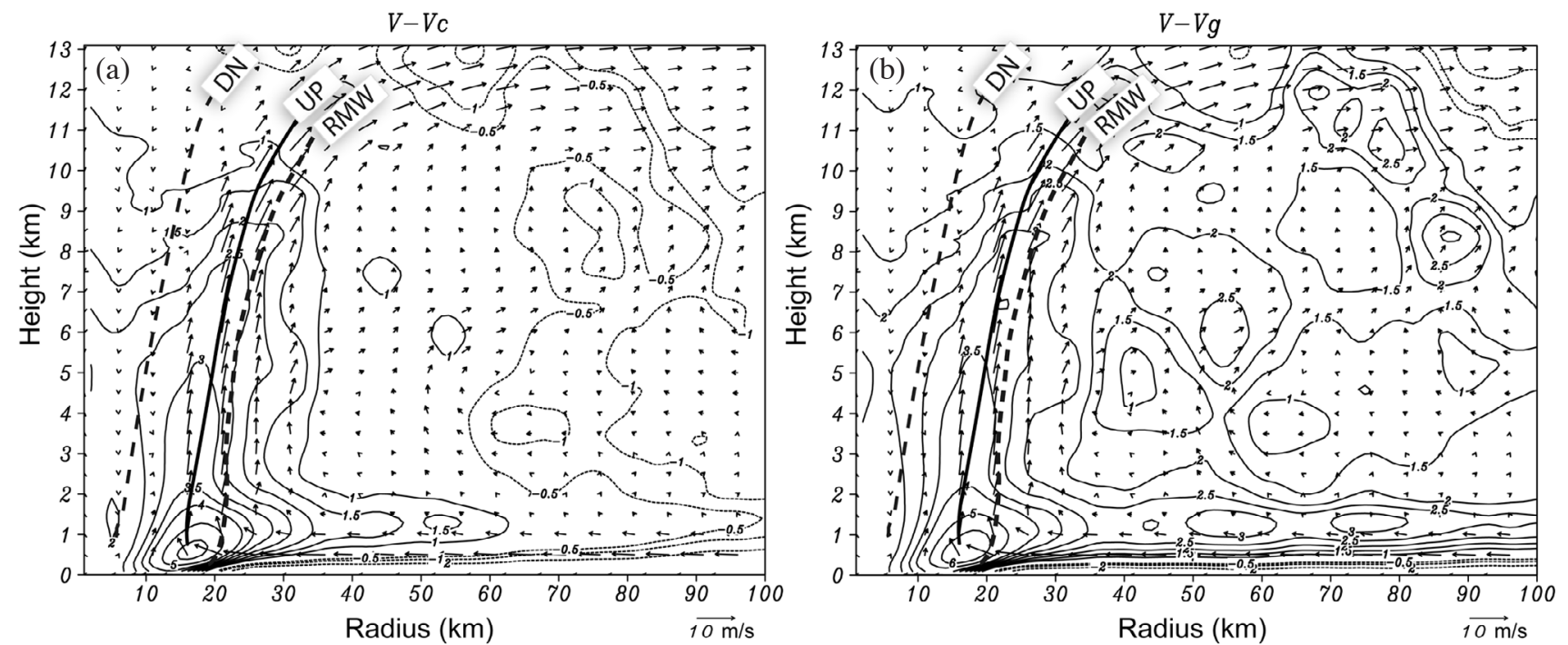

Fig. 7. As in Fig. 3 but for (a) acyclostrophic winds $\left(V-V_{C}\right)$, and (b) agradient winds $\left(V-V_{g}\right)$. (a) and (b) are both contoured at $-8,-6,-2,-1,-0.5$, $1,1.5,2,2.5,3,3.5,4,5,6$, and $8 \mathrm{~m} \mathrm{~s}^{-1}$. 

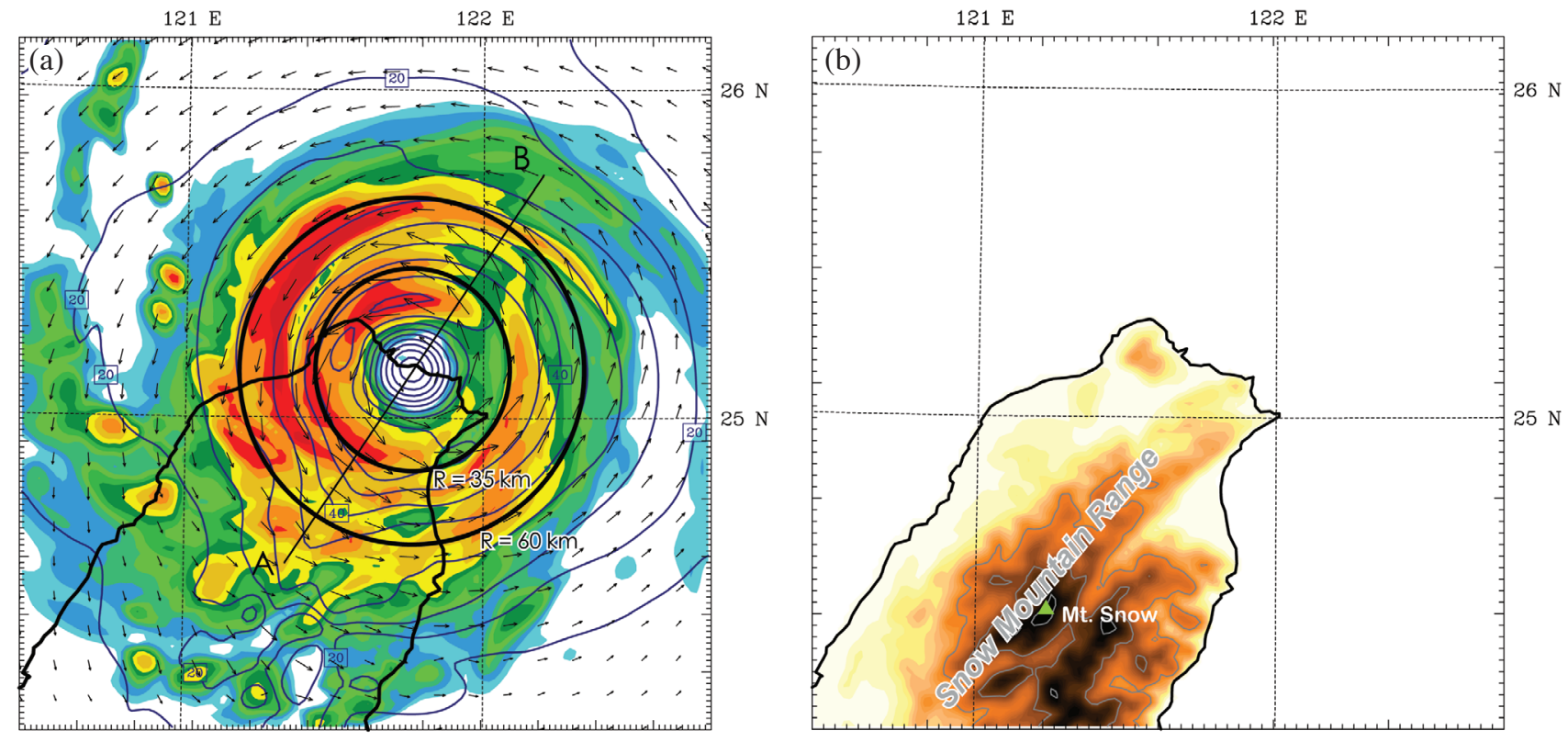

$\begin{array}{lllllllllllllllll}20 & 22 & 24 & 26 & 28 & 30 & 32 & 34 & 36 & 38 & 40 & 42 & 44 & 46 & 48 & 50 & \mathrm{dBZ}\end{array}$

$\begin{array}{lllllllllllllllllll}0 & 200 & 400 & 600 & 800 & 1000 & 1200 & 1400 & 1600 & 1800 & 2000 & 2200 & 2400 & 2600 & 2800 & 3000 & 3200 & \mathrm{~m}\end{array}$

Fig. 8. (a) Horizontal cross section of storm-relative tangential wind (dark-blue solid line contoured at $5 \mathrm{~m} \mathrm{~s}^{-1}$ ) at the 3 -km height taken from the 22 - $23 \mathrm{~h}$ integration or the period of $1000-1100$ UTC 16 September 2001. Superposed is the mid-level-mean $(\sigma=0.4-0.8)$ radar reflectivity and horizontal wind vector from the 2-km grid at 1030 UTC $(t=22.5 \mathrm{~h})$. Line AB shows the horizontal position of the vertical cross section in Fig. 9, and thick-solid circles show the rings of $r=35 \mathrm{~km}$ and $r=60 \mathrm{~km}$. (b) Color indicates the terrain height with extra contours at 1000, 2000, and $3000 \mathrm{~m}$.
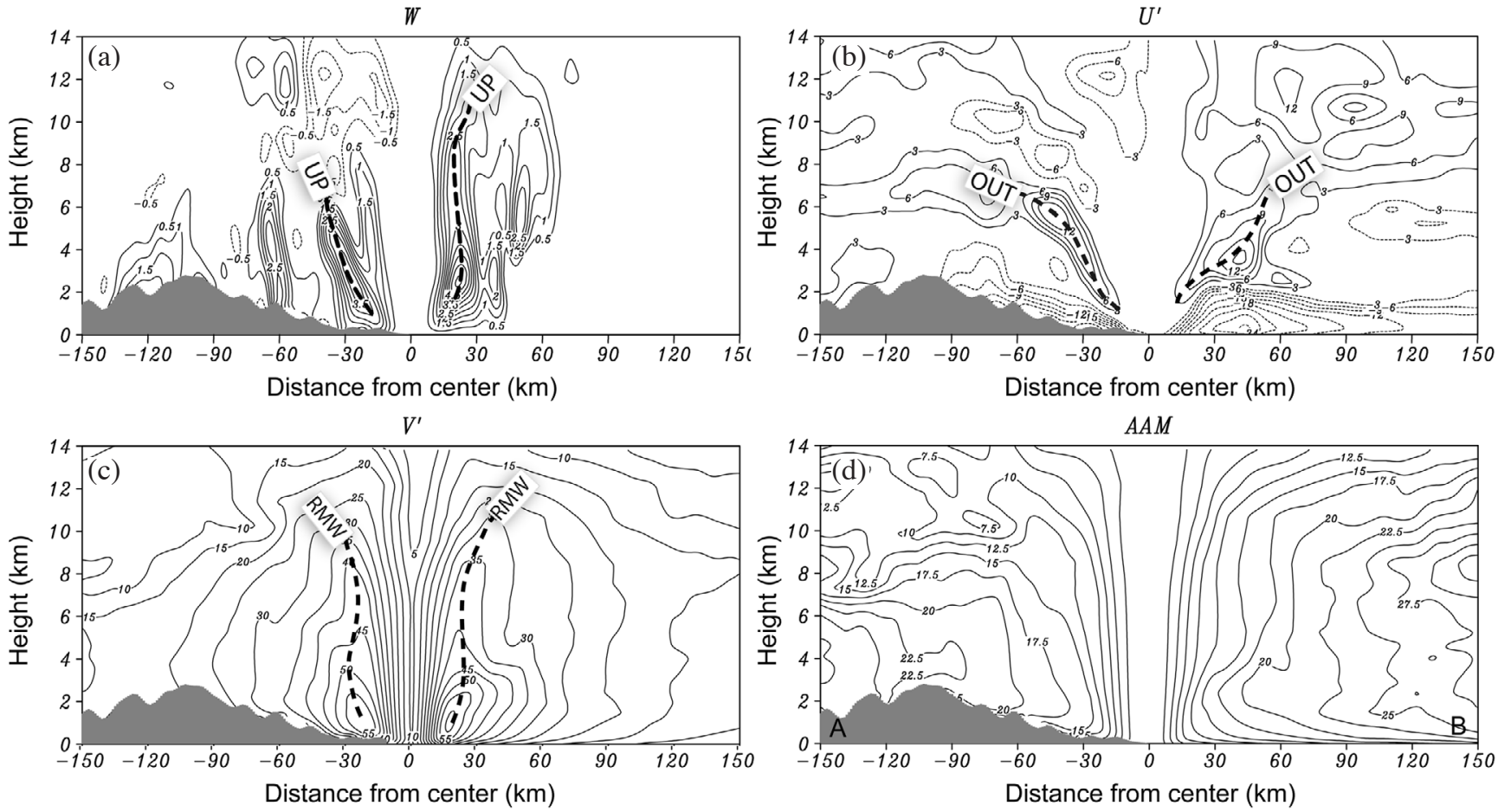

Fig. 9. As in Fig. 2 but for the NE-SW (AB) vertical cross section taken from the $22-23 \mathrm{~h}$ integration or the period of $1000-1100$ UTC 16 September 2001 (the horizontal position of $\mathrm{AB}$ cross section is in Fig. 8a). (a) is contoured at $0.5 \mathrm{~m} \mathrm{~s}^{-1}$; (b) is contoured at $3 \mathrm{~m} \mathrm{~s}^{-1}$; (c) is contoured at $5 \mathrm{~m} \mathrm{~s}^{-1}$; and (d) is contoured at $2.5 \times 10^{5} \mathrm{~m}^{2} \mathrm{~s}^{-1} \mathrm{~m} \mathrm{~s}^{-1}$. Horizontal winds at (b) and (c) are storm-relative, and zero contours in (a) and (b) are omitted. The radius of maximum wind, the axes of the eyewall updrafts and the midlevel outflow jet are represented by RMW, UP, and OUT, respectively. 

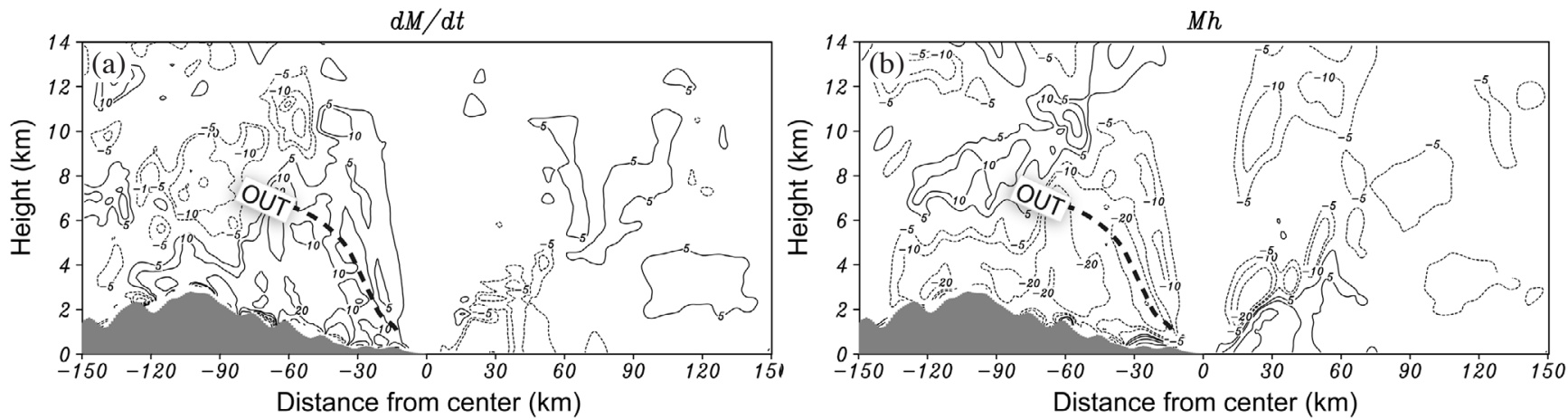

$M v$
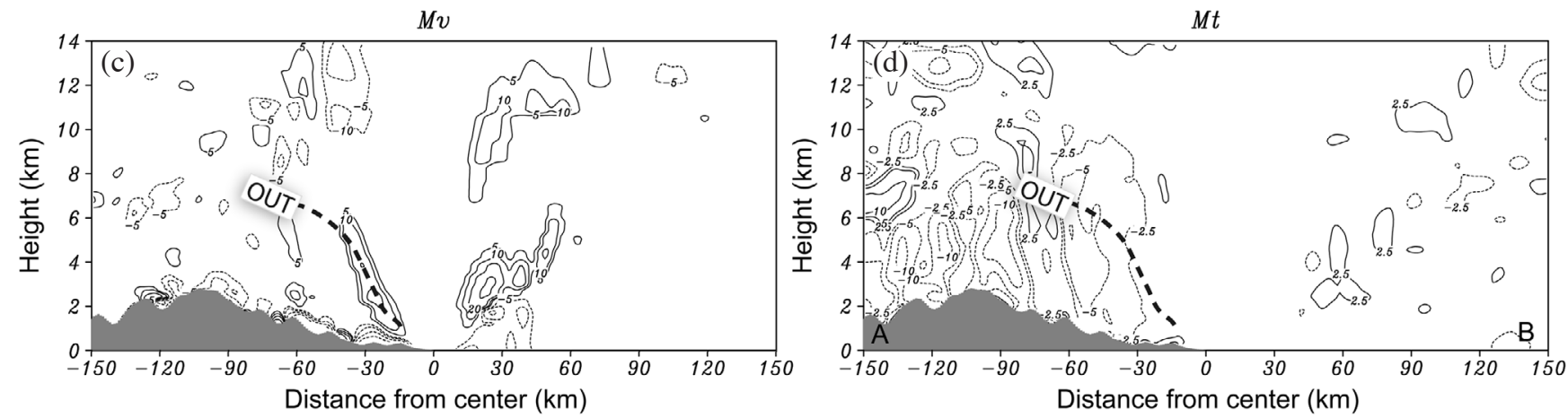

Fig. 10. Hourly-averaged AAM budget of Typhooh Nari along the NE-SW (AB) vertical cross section: (a) the net Lagrangian tendency due to all the sources/sinks $(d M / d t)$, (b) the horizontal advection, (c) the vertical advection, and (d) the local tendency. (a), (b) and (c) are contoured at \pm 5 , $\pm 10, \pm 20$, and $\pm 40 \times 10^{5} \mathrm{~m}^{2} \mathrm{~s}^{-1} \mathrm{~h}^{-1} ;$ (d) is contoured at $\pm 2.5, \pm 5$, and $\pm 10 \times 10^{5} \mathrm{~m}^{2} \mathrm{~s}^{-1} \mathrm{~h}^{-1}$. The axis of the outflow jet above terrain in (b) is represented by OUT.

are found over land, due to the positive pressure torque induced by tangential winds over terrain (Fig. 10a). The horizontal advection of the AAM is largely out of phase with the vertical advection of the AAM, particularly inside the eyewall (Figs. 10b, c). Because of enhanced surface friction and turbulent mixing over the terrain, the local tendency of AAM shows mostly negative (and some positive) accelerations over land; conversely, the local AAM tendency over the ocean is very small, again indicating the near conservation of the AAM (Fig. 10d).

During the first one hour after Nari's landfall on Taiwan, the radial flows have pronounced changes above terrain (cf. Figs. 2b and 9b), and the budget terms in Fig. 11 are used to discuss the physical mechanisms responsible for the changes of radial flows. The negative $\operatorname{PGF}_{\mathrm{R}}\left(U_{P}\right)$ still generates inward acceleration with a smaller magnitude at an increasing height; its peak value is decreased $\left(-450 \mathrm{~m} \mathrm{~s}^{-1} \mathrm{~h}^{-1}\right.$ over land vs. $-500 \mathrm{~m} \mathrm{~s}^{-1} \mathrm{~h}^{-1}$ over ocean) as a result of the weaker storm intensity after landfall (Fig. 11a). The centrifugal force $\left(U_{E}\right)$ shows a larger magnitude off shore $\left(>800 \mathrm{~m} \mathrm{~s}^{-1} \mathrm{~h}^{-1}\right)$ than that over land $\left(>500 \mathrm{~m} \mathrm{~s}^{-1} \mathrm{~h}^{-1}\right)$, due to stronger tangential winds off shore (Figs. 9c and 11b). Strong supercyclostrophic acceleration $\left(U_{P E}>0\right)$ occurs inside the eyewall off shore, and a sloping pattern of weak supercyclostrophic acceleration below the radial outflow jet (labeled by OUT) is found over land (Fig. 11c). The gradient wind imbalance $\left(U_{P E C}\right)$ pattern is still very similar to that of cyclostrophic force imbalance $\left(U_{P E}\right)$, even after the storm's landfall (Fig. 11d). Supergradient accelerations $\left(U_{P E C}>0\right)$ are evident for the flow above the MBL inside the eyewall off shore, because of a much larger centrifugal force associated with strong tangential wind over the $\mathrm{PGF}_{\mathrm{R}}$ within the ocean eyewall (Fig. 11d). Over land, the outward-tilted pattern of supergradient acceleration is right beneath the axis of the midlevel radial outflow (cf. Figs. 9b and 11d). Figure 11e shows mostly negative radial accelerations by turbulent mixing and diffusion inside the eyewall $(r<30 \mathrm{~km})$ off shore, reducing the radial outflows at the middle-to-upper levels. Weak positive radial accelerations by friction occur near the surface over land, leading to the slowdown of the low-level inflows; oscillations of positive and negative radial accelerations are found at the middleto-upper levels above terrain, due to the enhanced turbulent mixing associated with terrain-induced updrafts and downdrafts (Fig. 9a). The net Lagrangian acceleration in Fig. 11f displays a positive (radially outward) acceleration beneath the midlevel radial outflow over land, and also intense positive acceleration at the low-to-middle levels inside the eyewall off shore.

In order to understand the physical processes responsible for the net Lagrangian radial acceleration, Fig. 12 shows the associated horizontal advection, vertical advection, and 

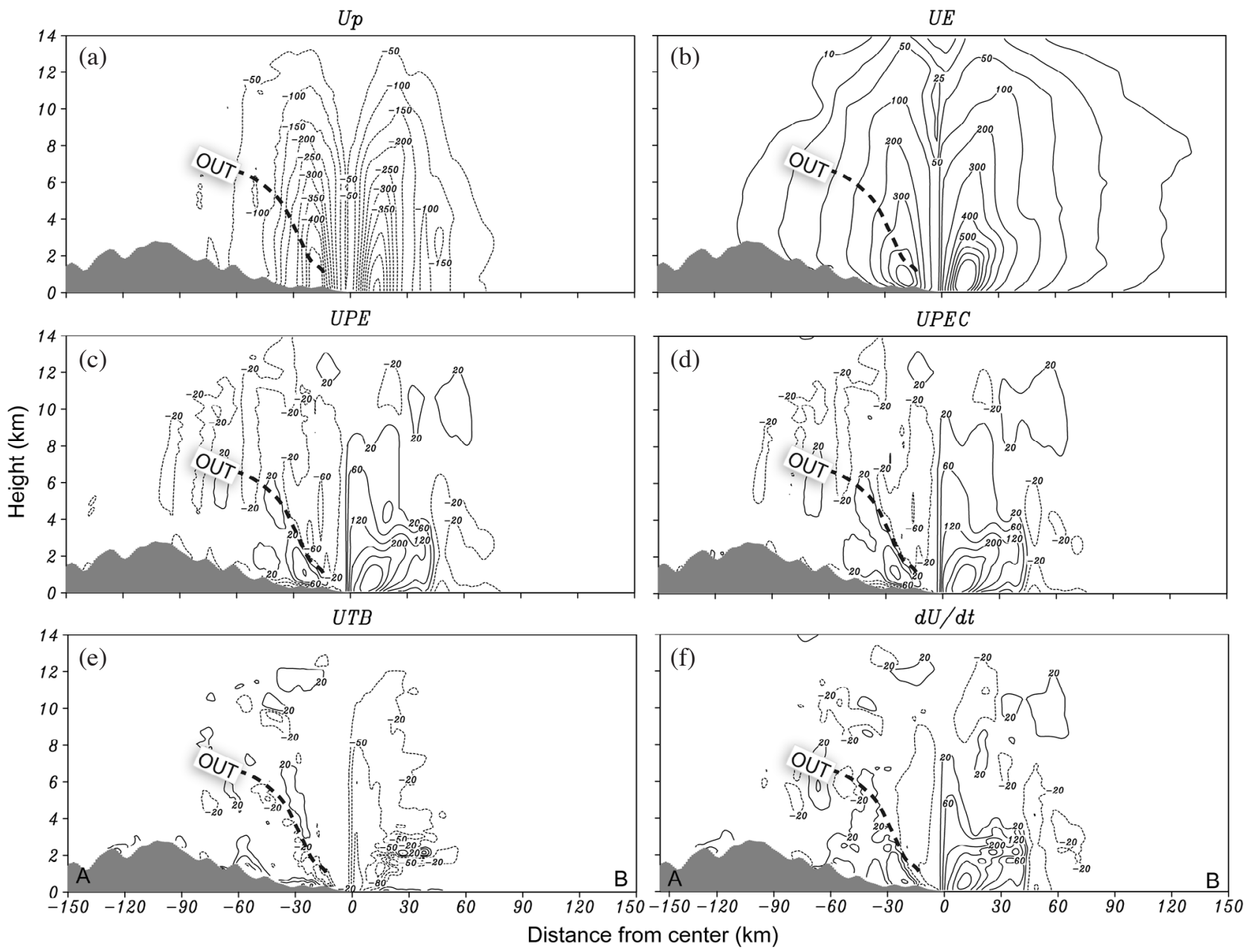

Fig. 11. As in Fig. 5 but for the NE-SW (AB) vertical cross section taken from the $22-23 \mathrm{~h}$ integration or the period of 1000 - 1100 UTC 16 September 2001. (a) is contoured at $50 \mathrm{~m} \mathrm{~s}^{-1} \mathrm{~h}^{-1}$; (b) is contoured at intervals of $100 \mathrm{~m} \mathrm{~s}^{-1} \mathrm{~h}^{-1}$ with extra contours at 10,25 , and $50 \mathrm{~m} \mathrm{~s}^{-1} \mathrm{~h}^{-1}$; (c) and (d) are contoured at $\pm 20, \pm 60,120,200$, and $300 \mathrm{~m} \mathrm{~s}^{-1} \mathrm{~h}^{-1}$; (e) is contoured at $\pm 20, \pm 50$ and $\pm 80 \mathrm{~m} \mathrm{~s}^{-1} \mathrm{~h}^{-1}$.
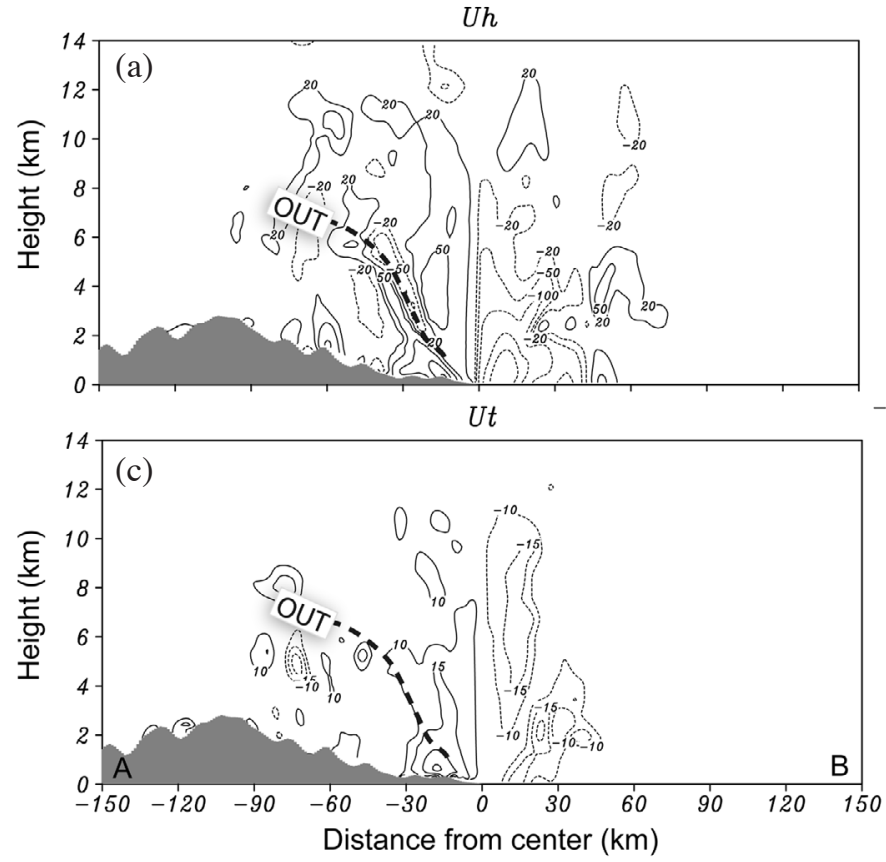

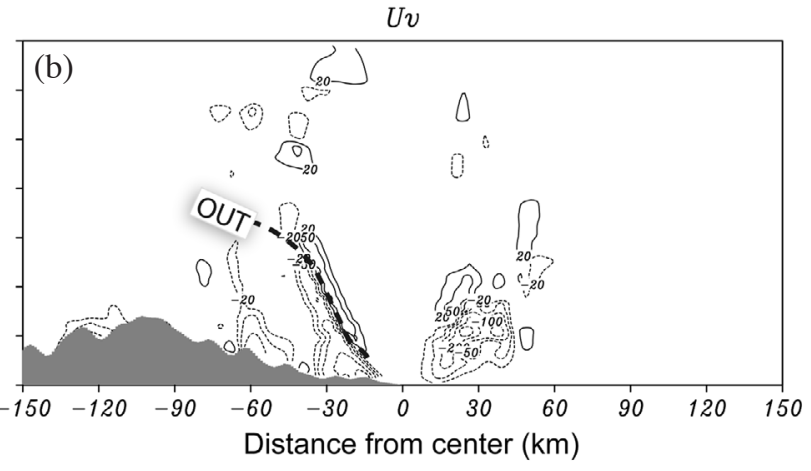

Fig. 12. As in Fig. 11, but for (a) horizontal advection, (b) vertical advection, and (c) local tendency for radial momentum. (a) and (b) are contoured at $\pm 20, \pm 50$ and $\pm 100 \mathrm{~m} \mathrm{~s}^{-1} \mathrm{~h}^{-1}$, while (c) is contoured at $\pm 10, \pm 15$ and $\pm 20 \mathrm{~m} \mathrm{~s}^{-1} \mathrm{~h}^{-1}$. 
local tendency, respectively. The vertical advection produces a negative acceleration below the outflow jet and positive acceleration above the jet inside the eyewall over terrain (Fig. 12b). On the other hand, the horizontal advection generates a reverse pattern, that is, positive acceleration below the outflow jet and negative acceleration above the jet over terrain (Fig. 12a). The local tendency is again one order of magnitude smaller than the horizontal and vertical advections (Fig. 12c), and shows an increase of negative (radially inward) acceleration in the inner core over ocean and an increase of positive (radially outward) acceleration in the inner core over land. Comparing to Fig. 12c with Fig. 11d, it is clear that the local positive acceleration inside the eyewall below the radial outflow jet over land is supergradient, due to the fact that the centrifugal force is stronger than the pressure-gradient and Coriolis forces.

Vertical distributions of radial wind, the Lagrangian tendency, and agradient (tangential) wind are shown in Fig. 13. It is evident from Figs. $12 \mathrm{~b}$ and 13a that the outward sloping radial outflow over land is accompanied by the vertical transport of positive (radially outward) Lagrangian acceleration (below the outflow jet). For the radial wind over the ocean, negative (radially inward) Lagrangian acceleration is responsible for the radial wind within the MBL for the outer region $(r>40 \mathrm{~km})$, and positive Lagrangian acceleration acts to slow down the MBL radial inflow inside the eyewall $(r<40 \mathrm{~km})$. Strong supergradient winds of greater than $15 \mathrm{~m} \mathrm{~s}^{-1}$ occur at a low level inside the eyewall

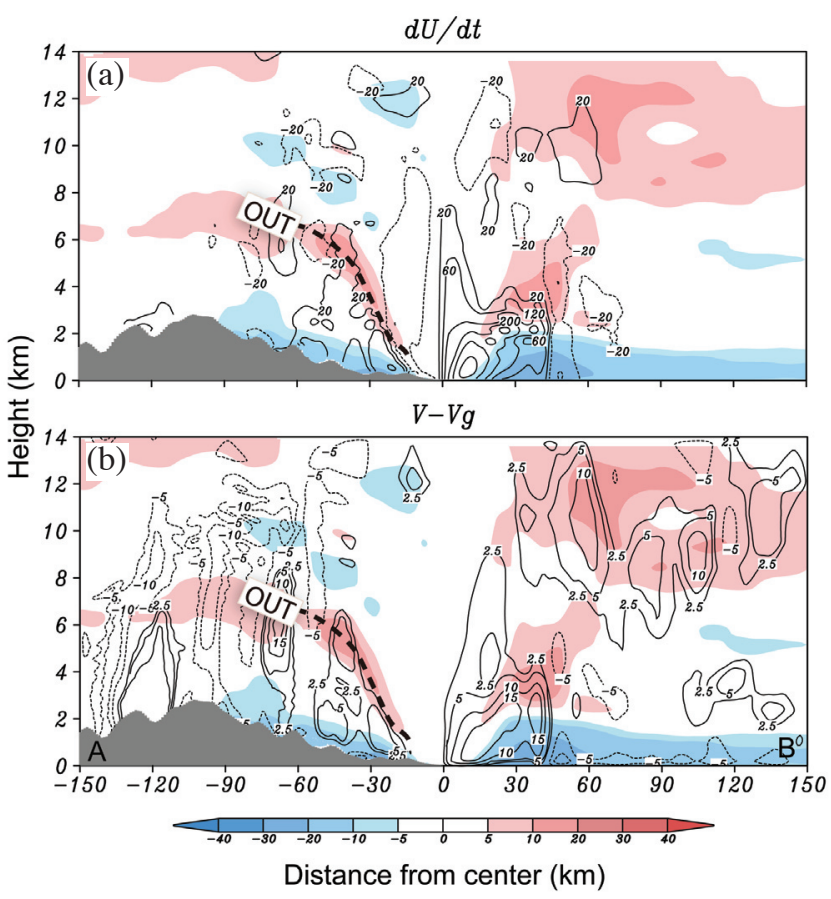

Fig. 13. As in Fig. 11 but for (a) Lagrangian tendency ( $d U / d t$ ), (b) agradient winds $\left(V-V_{g}\right.$, contoured at intervals of $5 \mathrm{~m} \mathrm{~s}^{-1}$ with an extra contour of $\left.2.5 \mathrm{~m} \mathrm{~s}^{-1}\right)$. Storm-relative radial winds are colored. off shore, slightly weaker supergradient winds $\left(>10 \mathrm{~m} \mathrm{~s}^{-1}\right)$ are in the upper-level outflow layer, and oscillations of supergradient and subgradient winds are found above the CMR (Fig. 13b). In particular, strong supergradient winds $\left(>5 \mathrm{~m} \mathrm{~s}^{-1}\right)$ are found within the outward-sloping radial outflow jet inside the eyewall over land.

Figure 14 shows the vertical-azimuthal distribution of temporally averaged radial-momentum budget terms at the radius of $35 \mathrm{~km}$ (near the eyewall with large gradient-wind imbalance; see Fig. 11d). Strong PGF $_{R}$ occurs at the low levels with a much weaker magnitude above (Fig. 14a), and intense centrifugal force associated with the strong tangential wind is found near 2-km height (Fig. 14b). The centrifugal force overcompensates the $\mathrm{PGF}_{\mathrm{R}}$ at most of the lower levels, leading to supercyclostrophic acceleration below $4 \mathrm{~km}$ (Fig. 14c). Owing to the small magnitude of the Coriolis acceleration, the pattern of the agradient acceleration is very similar to the acyclostrophic acceleration (Fig. 14d). The turbulent mixing produces positive and negative radialaccelerations above the terrain and near the level of strong tangential wind (near 2-km height; see Figs. 14b, e). The boundary layer effect generates positive radial-accelerations to slow down the low-level inflows, and this PBL-induced positive acceleration is weaker in the northern semicircle (mostly over ocean; see Fig. 8a) as a result of weaker surface friction over the ocean (Fig. 14e). The Lagrangian acceleration for radial momentum has a similar spatial distribution to that of the gradient-wind imbalance (Figs. 14d, f), except for more small-scale fluctuations associated with turbulent mixing.

At the outer region with a principal rainband to the west and north (i.e., $r=60 \mathrm{~km}$; see Fig. 8a), the radialmomentum budget terms have a similar vertical-azimuthal distribution to those near the eyewall (Figs. 14a, b) but with much weaker magnitudes (Figs. 15a, b). The $\mathrm{PGF}_{\mathrm{R}}$ is stronger than the centrifugal force for most of vertical levels at $r$ $=60 \mathrm{~km}$ except for above the terrain and over the northwestern quadrant where an intense rainband is located (Fig. 8a), leading to mostly subcyclostrophic accelerations (Fig. 15c). With the inclusion of the small positive Coriolis acceleration, the pattern of the gradient wind imbalance is still similar to the cyclostrophic force imbalance, but with a slightly more positive acceleration (Fig. 15d). The turbulent mixing, again, produces positive and negative radial-accelerations, particularly above the terrain, and the boundary layer effect generates positive accelerations to retard the radial inflow (Fig. 15e). The Lagrangian acceleration for the radial momentum, again, has a similar spatial distribution to that of the gradient-wind imbalance (Figs. 15d, f).

Figure 16 illustrates the vertical-azimuthal distributions of the agradient (tangential) winds at the radii of 35 and $60 \mathrm{~km}$, respectively. Near the eyewall (at the $35-\mathrm{km}$ radius; Fig. 16a), strong supergradient winds (with a peak magnitude of $21 \mathrm{~m} \mathrm{~s}^{-1}$ at 2-km altitude) occur at low levels 
in the northern semicircle and strong subgradient winds (with a peak value of $-24 \mathrm{~m} \mathrm{~s}^{-1}$ ) are found below $2 \mathrm{~km}$ in the southeastern quadrant (on the lee side of SMR). Supergradient winds up to $9 \mathrm{~m} \mathrm{~s}^{-1}$ also occur above the terrain at the low-to-middle levels. Over the outer region (at the $60-\mathrm{km}$ radius; Fig. 16b), strong subgradient winds (with a peak magnitude of $24 \mathrm{~m} \mathrm{~s}^{-1}$ near the surface) still occur below $2 \mathrm{~km}$ on the lee side (also in the southeastern quadrant), supergradient winds occur within the whole troposphere in the northwestern quadrant (where an intense rainband was located; see Fig. 8a), and supergradient winds up to $6 \mathrm{~m} \mathrm{~s}^{-1}$ are found above the terrain at the midlevel (Fig. 16b).

\section{CONCLUSIONS}

In this paper, we have investigated the development of gradient-wind unbalanced accelerations and winds within the vortex circulation of Typhoon Nari, as the storm moves
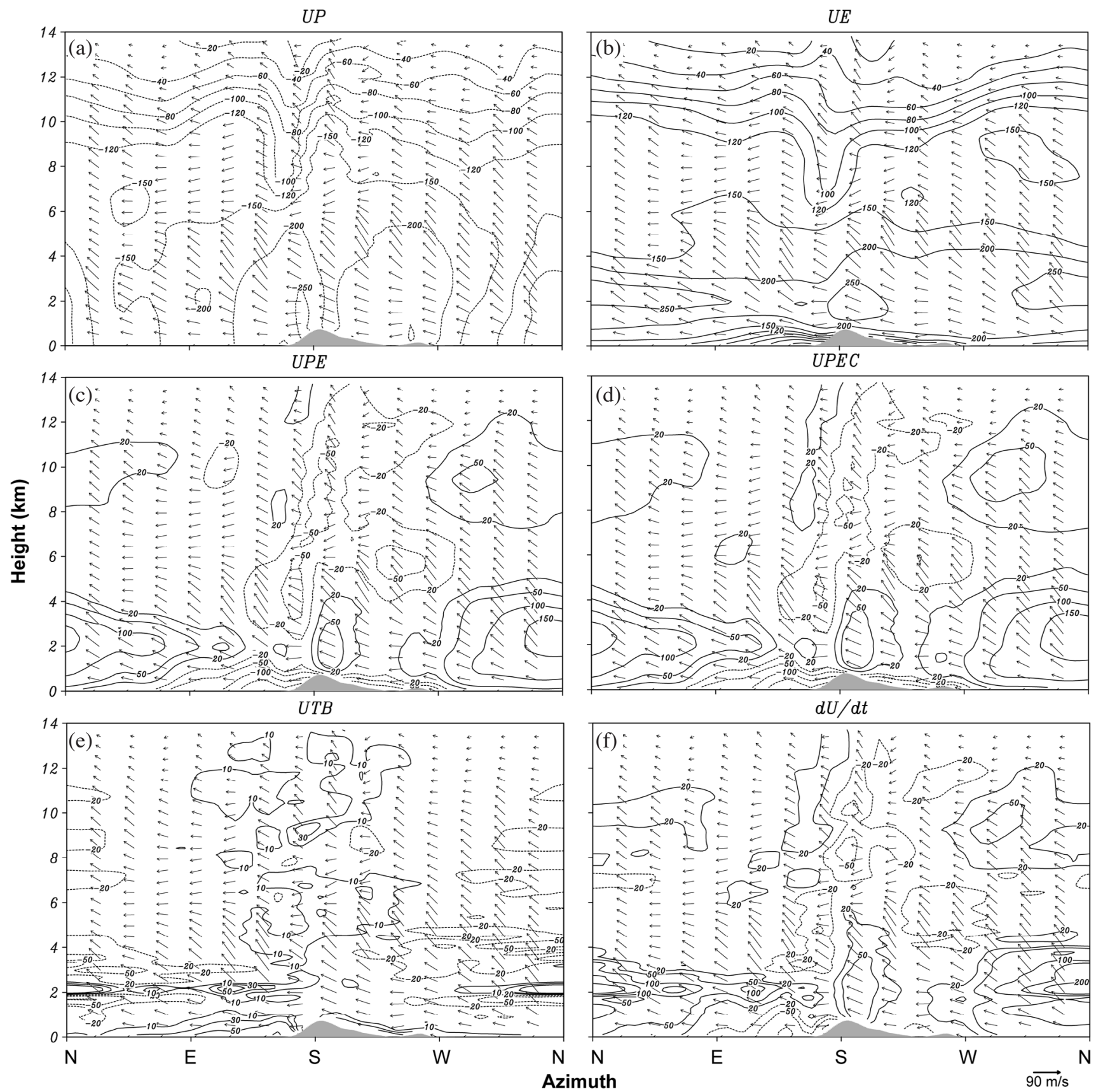

Fig. 14. As in Fig. 11 but for the circle of $r=35 \mathrm{~km}$ taken from the $22-23 \mathrm{~h}$ integration or the period of $1000-1100$ UTC 16 September 2001. Superposed are the azimuth-height flow vectors. (a) and (b) are contoured at $\pm 20, \pm 40, \pm 60, \pm 80, \pm 100, \pm 120, \pm 150, \pm 200$, and $\pm 250 \mathrm{~m} \mathrm{~s}^{-1} \mathrm{~h}^{-1}$; (c), (d) and (f) are contoured at $\pm 20, \pm 50, \pm 100, \pm 150$, and $\pm 200 \mathrm{~m} \mathrm{~s}^{-1} \mathrm{~h}^{-1}$; (e) is contoured at $-50,-20,10,30$, and $50 \mathrm{~m} \mathrm{~s}^{-1} \mathrm{~h}^{-1}$. 
from the ocean to northern Taiwan, through analyses of the AAM and radial momentum budgets. The high-resolution model output (with 2-km horizontal grid spacing and 2-min output interval) from Part I is used to calculate the budgets. To our knowledge, this is the first study to document the evolution of tangential and radial flows of a TC during the landfall process and to examine the physical mechanism responsible for the sloping midlevel radial outflow jet above steep terrain. Our findings are summarized below.
It was found from the AAM budgets that AAM was nearly conserved outside the eyewall and above the MBL for Nari at the oceanic phase; the MBL was the major sink of the AAM because frictional dissipation and turbulent mixing decreased the local AAM inside the eyewall. After the storm's landfall on northern Taiwan, the strong tangential winds produced a positive pressure torque over the terrain, resulting in positive Largrangian tendencies of the AAM and the increase of tangential winds above the SMR.
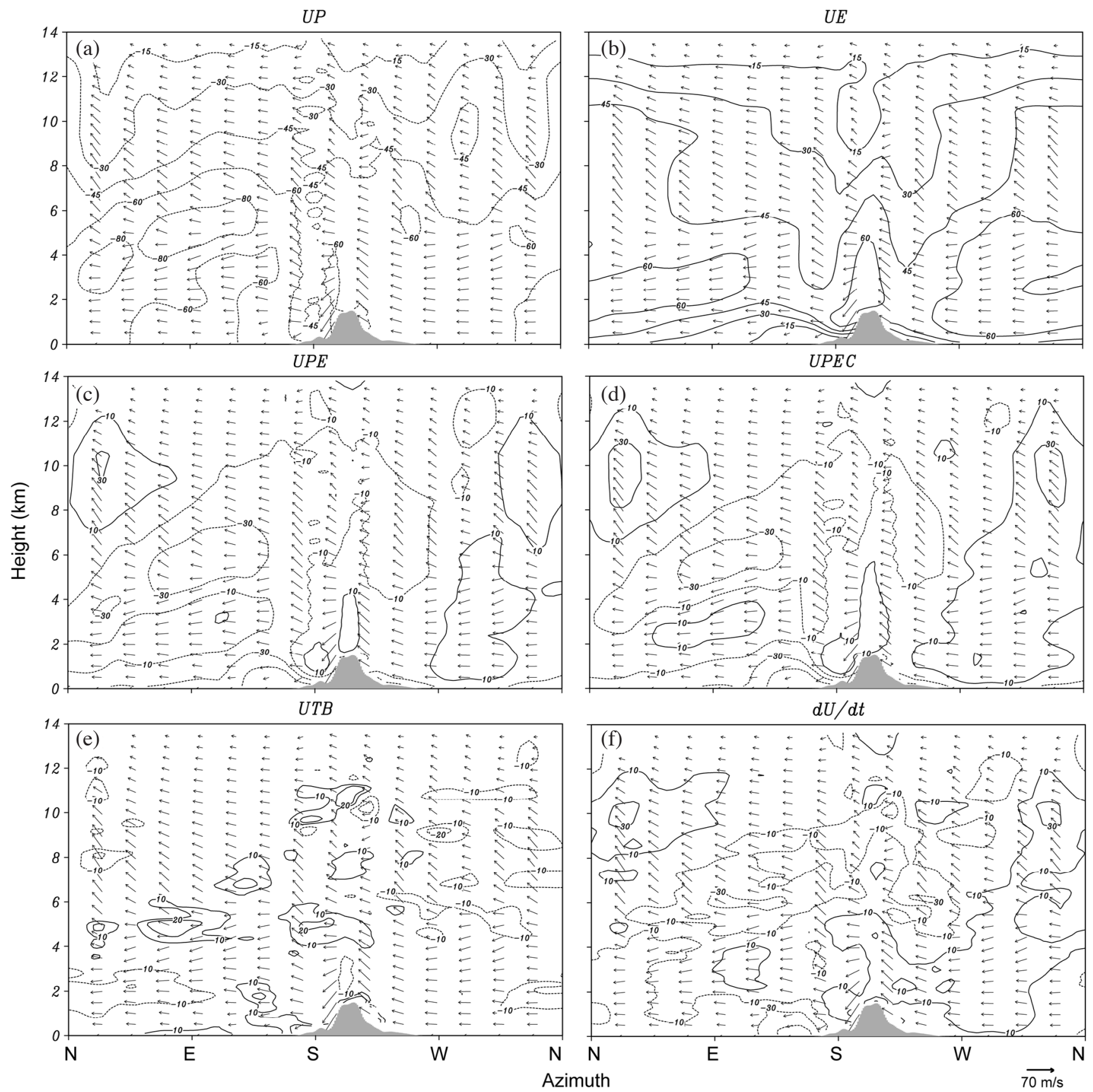

Fig. 15. As in Fig. 14 but for the circle of $r=60 \mathrm{~km}$. (a) and (b) are contoured at $\pm 15, \pm 30, \pm 45, \pm 60, \pm 80$ and $\pm 100 \mathrm{~m} \mathrm{~s}^{-1} \mathrm{~h}^{-1}$; (c), (d) and (f) are contoured at $\pm 10, \pm 30, \pm 50, \pm 100$, and $\pm 200 \mathrm{~m} \mathrm{~s}^{-1} \mathrm{~h}^{-1}$; (e) is contoured at $\pm 10, \pm 20$, and $\pm 50 \mathrm{~m} \mathrm{~s}^{-1} \mathrm{~h}^{-1}$. 


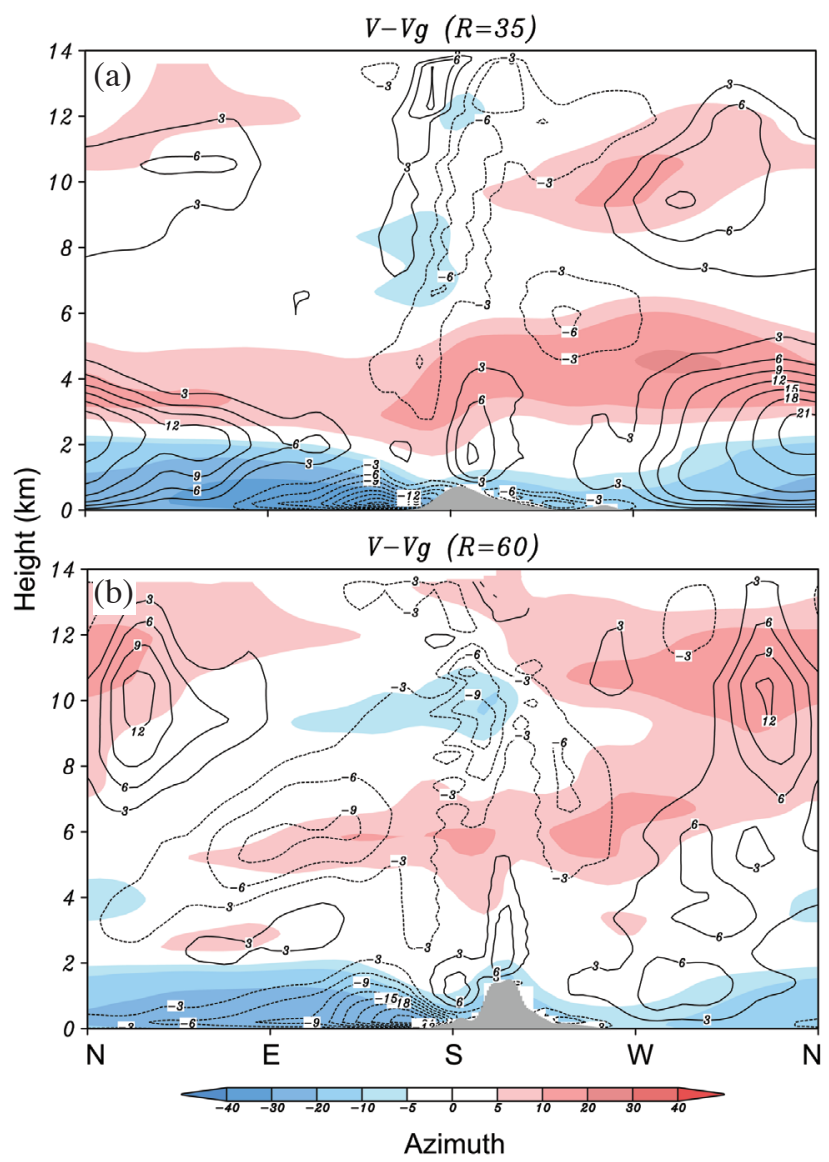

Fig. 16. Vertical cross sections of agradient winds $\left(V-V_{g}\right.$; contoured at $3 \mathrm{~m} \mathrm{~s}^{-1}$ ) for the circles of (a) $r=35 \mathrm{~km}$ and (b) $r=60 \mathrm{~km}$ taken from the 22 - $23 \mathrm{~h}$ integration or the period of 1000 - 1100 UTC 16 September 2001. Storm-relative radial winds are colored.

The vertical advection of the AAM was largely out of phase with the horizontal advection of the AAM, and the enhanced surface friction and turbulent mixing over land produced mostly negative local tendencies of the AAM.

The radial momentum budget indicates that supergradient accelerations and flows are pronounced from the bottom of the eye to the upper outflow layer inside the eyewall for the oceanic storm, even after being temporally and azimuthally averaged. These strong supergradient accelerations and flows result from much larger centrifugal force associated with strong tangential wind inside the eyewall relative to the radial pressure-gradient force. Subgradient accelerations and flows occurred below the MBL outside the eyewall. After Nari's landfall on Taiwan, both radar observation and model simulation indicate that the radial inflows at lower levels became thicker and stronger over land, and the sloping radial outflow jet was maximized at the midlevel above the rugged topography of Taiwan. Centrifugal and Coriolis forces overcompensated the pressure-gradient force, resulting in supergradient accelerations; the vertical transport of supergradient accelerations by strong updrafts inside the eyewall above the terrain produced the sloping midlevel radial outflows. Enhanced surface friction over land acted to slowdown the low-level inflows. Stronger turbulent mixing associated with terrain-induced updrafts/downdrafts generated oscillations of positive and negative radial accelerations above the topography.

For the oceanic storm, the gradient wind balance may be a good approximation for tangential wind (particularly for the axisymmetric component) above the MBL and below the upper outflow layer, and the maximum supergradient wind up to $6 \mathrm{~m} \mathrm{~s}^{-1}$ occurred near the top of MBL inside the eyewall. After the storm's landfall, the enhanced turbulent mixing above rugged terrain and stronger surface friction over land produced a stronger gradient-wind imbalance, indicating that the GWB model is no longer appropriate to describe the tangential winds over terrain, even at middle levels. Near the eyewall, a supergradient wind can be as strong as $9 \mathrm{~m} \mathrm{~s}^{-1}$ above the terrain and subgradient wind up to $-21 \mathrm{~m} \mathrm{~s}^{-1}$ is found at the surface on the lee side. In the outer region, agradient winds remain similar spatial patterns relative to Taiwan's topography (supergradient winds above the terrain and subgradient winds on the lee side), despite weaker magnitudes. The outward-sloping midlevel outflow jet above terrain consists mainly of these supergradient winds of $5-9 \mathrm{~m} \mathrm{~s}^{-1}$.

When Nari is still over the ocean and intensifying, the local changes of the AAM and radial momentum are very small due to the quasi-balanced nature of storm dynamics. After Nari's landfall in northern Taiwan, the enhanced force imbalances produce larger local changes of the AAM and radial momentum, leading to more quickly-evolved vortex flows and secondary circulations above Taiwan's steep terrain.

It should be noted that the above results are obtained primarily from analyses of model simulation of a landfall typhoon and some model deficiencies still remain, such as the imperfect initial and boundary conditions, the use of 2-km grid size (which is still too coarse to explicitly resolve deep convection and turbulence above rugged terrain), and some uncertainties in physical parameterizations. Nevertheless, much agreement between the simulation and observation shown in Part I and Fig. 1 suggests that the results presented herein are relevant to real landfall typhoons. Similar evolution of the AAM and radial-momentum budgets might have also occurred in Typhoon Morakot (2009) as it came across Taiwan and produced heavy rainfall (Hendricks et al. 2011; Huang et al. 2011; Tao et al. 2011; Yen et al. 2011).

Acknowledgements We appreciate two reviewers' helpful comments which improved the quality of this manuscript significantly. This work was supported by the National Science Council of Taiwan under grant No. NSC 97-2111-M008-019-MY3, NSC 99-2625-M-008-005-MY3, and NSC 98-2745-M-008-012-MY3. Yi Zhang was supported by the 
National Natural Science Foundation of China under grant No. 40605017, 40921160382, and the National Special Funding Project for Meteorology (GYHY201006004).

\section{REFERENCES}

Dudhia, J., 1993: A nonhydrostatic version of the Penn State-NCAR mesoscale model: Validation tests and simulation of an Atlantic cyclone and cold front. Mon. Weather Rev., 121, 1493-1513, doi: 10.1175/1520-049 3(1993)121<1493:ANVOTP>2.0.CO;2. [Link]

Emanuel, K. A., 1986: An air-sea interaction theory for tropical cyclones. Part I: Steady-state maintenance. $J$. Atmos. Sci., 43, 585-605, doi: 10.1175/1520-0469(19 86)043<0585:AASITF>2.0.CO;2. [Link]

Gray, W. M. and D. J. Shea, 1973: The hurricane's inner core region. II. Thermal stability and dynamic characteristics. J. Atmos. Sci., 30, 1565-1576, doi: 10.1175/1 520-0469(1973)030<1565:THICRI > 2.0.CO;2. [Link]

Grell, G. A., J. Dudhia, and D. R. Stauffer, 1995: A description of the fifth-generation Penn State/NCAR Mesoscale Model. NCAR Technical Note, 138 pp.

Hendricks, E. A., J. R. Moskaitis, Y. Jin, R. M. Hodur, J. D. Doyle, and M. S. Peng, 2011: Prediction and diagnosis of Typhoon Morakot (2009) using the Naval Research Laboratory's mesoscale tropical cyclone model. Terr. Atmos. Ocean. Sci., 22, 579-594, doi: 10.3319/ TAO.2011.05.30.01(TM). [Link]

Huang, C. Y., C. S. Wong, and T. C. Yeh, 2011: Extreme rainfall mechanisms exhibited by Typhoon Morakot (2009). Terr. Atmos. Ocean. Sci., 22, 613-632, doi: 10.3319/TAO.2011.07.01.01(TM). [Link]

Kurihara, Y. and M. A. Bender, 1982: Structure and analysis of the eye of a numerically simulated tropical cyclone. J. Meteorol. Soc. Jpn., 60, 381-395.

La Seur, N. E. and H. F. Hawkins, 1963: An analysis of Hurricane Cleo (1958) based on data from research reconnaissance aircraft. Mon. Weather Rev., 91, 694-709, doi: 10.1175/1520-0493(1963)091<0694:AAOHCB> 2.3.CO;2. [Link]

Li, M. H., M. J. Yang, R. Soong, and H. L. Huang, 2005: Simulating typhoon floods with gauge data and mesoscale-modeled rainfall in a mountainous watershed. J. Hydrometeorol., 6, 306-323, doi: 10.1175/JHM423.1 . [Link]

Liu, Y., D. L. Zhang, and M. K. Yau, 1997: A multiscale numerical study of Hurricane Andrew (1992). Part I: Explicit simulation and verification. Mon. Weather Rev., 125, 3073-3093, doi: 10.1175/1520-0493(1997)125<3 073:AMNSOH>2.0.CO;2. [Link]

Liu, Y., D. L. Zhang, and M. K. Yau, 1999: A multiscale numerical study of Hurricane Andrew (1992). Part II: Kinematics and inner-core structures. Mon. Weather Rev., 127, 2597-2616, doi: 10.1175/1520-0493(1999)
127<2597:AMNSOH>2.0.CO;2. [Link]

Shapiro, L. J. and H. E. Willoughby, 1982: The response of balanced hurricanes to local sources of heat and momentum. J. Atmos. Sci., 39, 378-394, doi: 10.1175/152 0-0469(1982)039<0378:TROBHT>2.0.CO;2. [Link]

Sui, C. H., C. Y. Huang, Y. B. Tsai, C. S. Chen, P. L. Lin, S. L. Shieh, M. H. Li, Y. A. Liou, T. C. Chen Wang, R. S. Wu, G. R. Liu, and Y. H. Chu, 2002: Meteorology-hydrology study targets Typhoon Nari and Taipei flood. Eos, Trans., AGU, 83, 265-270, doi: 10.1029/ 2002EO000186. [Link]

Sui, C. H., X. Li, M. J. Yang, and H. L. Huang, 2005: Estimation of oceanic precipitation efficiency in cloud models. J. Atmos. Sci., 62, 4358-4370, doi: 10.1175/ JAS3587.1. [Link]

Tao, W. K., J. J. Shi, P. L. Lin, J. Chen, S. Lang, M. Y. Chang, M. J. Yang, C. C. Wu, C. Peters-Lidard, C. H. Sui, and B. J. D. Jou, 2011: High-resolution numerical simulation of the extreme rainfall associated with Typhoon Morakot. Part I: Comparing the impact of microphysics and PBL parameterizations with observations. Terr. Atmos. Ocean. Sci., 22, 673-696, doi: 10.3319/TAO.2011.08.26.01(TM). [Link]

Willoughby, H. E., 1990: Gradient balance in tropical cyclones. J. Atmos. Sci., 47, 265-274, doi: 10.1175/15200469(1990)047<0265:GBITC>2.0.CO;2. [Link]

Willoughby, H. E., 1991: Reply. J. Atmos. Sci., 48, 12091212, doi: 10.1175/1520-0469(1991)048<1209:R>2.0. $\mathrm{CO} ; 2$. [Link]

Yang, M. J. and R. A. Houze, 1996: Momentum budget of a squall line with trailing stratiform precipitation: Calculations with a high-resolution numerical model. J. Atmos. Sci., 53, 3629-3652, doi: 10.1175/1520-0469 (1996)053<3629:MBOASL>2.0.CO;2. [Link]

Yang, M. J., D. L. Zhang, and H. L. Huang, 2008: A modeling study of Typhoon Nari (2001) at landfall. Part I: Topographic effects. J. Atmos. Sci., 65, 3095-3115, doi: 10.1175/2008JAS2453.1. [Link]

Yang, M. J., D. L. Zhang, X. D. Tang, and Y.Zhang, 2011a: A modeling study of Typhoon Nari (2001) at landfall. Part II: Structural changes and terrain-induced asymmetries. J. Geophys. Res., 116, D09112, doi: 10.1029/ 2010JD015445. [Link]

Yang, M. J., S. A. Braun, and D. S. Chen, 2011b: Water budget of Typhoon Nari (2001). Mon. Weather Rev., in press.

Yeh, T.C. and R.L. Elsberry, 1993a: Interaction of typhoons with the Taiwan orography. Part I: Upstream track deflections. Mon. Weather Rev., 121, 3193-3212, doi: 10.1175/1520-0493(1993)121<3193:IOTWTT>2.0. CO;2. [Link]

Yeh, T. C. and R. L. Elsberry, 1993b: Interaction of typhoons with the Taiwan orography. Part II: Continuous and discontinuous tracks across the island. Mon. 
Weather Rev., 121, 3213-3233, doi: 10.1175/1520-04 93(1993)121<3213:IOTWTT>2.0.CO;2. [Link]

Yen, T. H., C. C. Wu, and G. Y. Lien, 2011: Rainfall simulations of Typhoon Morakot with controlled translation speed based on EnKF data assimilation. Terr. Atmos. Ocean. Sci., 22, 647-660, doi: 10.3319/TAO.2011.07.
05.01(TM). [Link]

Zhang, D. L., Y. Liu, and M. K. Yau, 2001: A multiscale numerical study of Hurricane Andrew (1992). Part IV: Unbalanced Flows. Mon. Weather Rev., 129, 92-107, doi: 10.1175/1520-0493(2001)129<0092:AMNSOH> 2.0.CO;2. [Link] 